\title{
Altered gene regulation and synaptic morphology in Drosophila learning and memory mutants
}

\author{
Zhuo Guan, Lauren K. Buhl, William G. Quinn, and J. Troy Littleton ${ }^{1}$ \\ Department of Biology and Department of Brain and Cognitive Sciences, The Picower Institute for Learning and Memory, \\ Massachusetts Institute of Technology, Cambridge, Massachusetts 02139, USA
}

\begin{abstract}
Genetic studies in Drosophila have revealed two separable long-term memory pathways defined as anesthesia-resistant memory (ARM) and long-lasting long-term memory (LLTM). ARM is disrupted in radish (rsh) mutants, whereas LLTM requires CREB-dependent protein synthesis. Although the downstream effectors of ARM and LLTM are distinct, pathways leading to these forms of memory may share the cAMP cascade critical for associative learning. Dunce, which encodes a cAMP-specific phosphodiesterase, and rutabaga, which encodes an adenylyl cyclase, both disrupt short-term memory. Amnesiac encodes a pituitary adenylyl cyclase-activating peptide homolog and is required for middle-term memory. Here, we demonstrate that the Radish protein localizes to the cytoplasm and nucleus and is a PKA phosphorylation target in vitro. To characterize how these plasticity pathways may manifest at the synaptic level, we assayed synaptic connectivity and performed an expression analysis to detect altered transcriptional networks in rutabaga, dunce, amnesiac, and radish mutants. All four mutants disrupt specific aspects of synaptic connectivity at larval neuromuscular junctions (NM)s). Genome-wide DNA microarray analysis revealed $\sim 375$ transcripts that are altered in these mutants, suggesting defects in multiple neuronal signaling pathways. In particular, the transcriptional target Lapsyn, which encodes a leucine-rich repeat cell adhesion protein, localizes to synapses and regulates synaptic growth. This analysis provides insights into the Radish-dependent ARM pathway and novel transcriptional targets that may contribute to memory processing in Drosophila.
\end{abstract}

[Supplemental material is available for this article.]

Genetic screens for behavioral mutants in Drosophila melanogaster have been instrumental in identifying the stages and molecular mechanisms underlying learning and memory. Studies of associative learning between olfactory cues and aversive electric shocks have led to the identification of mutants that disrupt distinct forms of information storage. Short-term memory (STM) is observed directly after training and is protein-synthesis independent, but can be blocked by agents that disrupt neural activity such as anesthetics. Long-term memory can be established via two distinct pathways: anesthesia-resistant memory (ARM) and longlasting long-term memory (LLTM). ARM forms immediately after training and can last for days depending on training intensity (Quinn and Dudai 1976). Unlike LLTM, however, reducing protein synthesis with the translation-inhibitor cycloheximide does not disrupt ARM (Tully et al. 1994). LLTM can last for up to a week in Drosophila and is induced by spaced training, in which training cycles are interrupted by rest intervals (Tully et al. 1994). Understanding the mechanisms that underlie these distinct stages of memory would provide insights into how information storage is achieved at a molecular level.

The cAMP-signaling pathway has been linked to STM formation in Drosophila, as the learning mutants dunce (dnc) and rutabaga (rut) are both defective in cAMP metabolism. The $d n c$ gene encodes a cAMP-specific phosphodiesterase (Dudai et al. 1976; Byers et al. 1981; Chen et al. 1986), whereas rut encodes an adenylyl cyclase (Livingstone et al. 1984; Krupinski et al. 1989; Levin et al. 1992). Both $d n c^{1}$ and $r u t^{1}$ mutants display

'Corresponding author.

E-mail troy@mit.edu; fax (617) 452-2249.

Article is online at http://www.learnmem.org/cgi/doi/10.1101/lm.2027111. abnormal synaptic morphology and physiology at the Drosophila neuromuscular junction (NMJ) (Renger et al. 2000), a wellcharacterized model synapse for assaying synaptic connectivity. $d n c^{1}$ mutants, which have elevated cAMP levels, show increased numbers of synaptic boutons and axonal branches along with increased neurotransmitter release (Zhong and Wu 1991; Zhong et al. 1992; Davis et al. 1996). Conversely, rut ${ }^{1}$ mutants that have reduced cAMP levels display opposite effects on synaptic structure and neurotransmission (Zhong and Wu 1991; Zhong et al. 1992), suggesting that abnormal regulation of synaptic connectivity and function may contribute to the learning defects in these mutants. Further implicating the cAMP pathway in memory formation is the amnesiac ( $\mathrm{amn}$ ) mutant, whose gene product encodes a neuropeptide with homology to mammalian pituitary adenylyl cyclase-activating peptide (PACAP) (Quinn et al. 1979; Feany and Quinn 1995; Moore et al. 1998; Waddell et al. 2000). Together, these and other studies have highlighted the role of the cAMP pathway as a key molecular player in learning events across multiple species.

The mechanisms underlying ARM and LLTM are less characterized, but several clues to their biological underpinnings have been identified. ARM in Drosophila requires the activity of the radish $(r s h)$ gene, whereas LLTM requires cAMP-dependent protein kinase (PKA) activation of CREB (cAMP response element binding protein) and subsequent protein synthesis. $r s h^{1}$ mutants are deficient in ARM but show normal LLTM, whereas transgenic flies that ectopically express an inhibitory isoform of CREB (CREB2b) show normal ARM but are deficient in LLTM (Yin et al. 1994), suggesting that ARM and LLTM involve different biochemical pathways. Inhibiting both CREB and the rsh pathway blocks all long-term memory in Drosophila. To date, the radish gene is the only 
molecular link to the poorly understood ARM phase of long-term memory. The $r$ sh gene has been identified as CG15720 by comparative sequencing of genomic DNA from $r s h^{1}$ and controls and is confirmed by phenotypic rescue (Folkers et al. 2006). $r$ sh encodes a novel serine/arginine-rich protein with 23 predicted PKA phosphorylation sites, but lacks other regions of homology to suggest a specific function. Radish has also been reported to bind Rac1 (Formstecher et al. 2005), a transducer protein implicated in an active mechanism of forgetting in Drosophila (Shuai et al. 2010). $r s h^{1}$ mutant flies show normal initial learning, but their memory rapidly decays within $8 \mathrm{~h}$ following training (Folkers et al. 1993). $r s h^{1}$ mutant flies that have been anesthetized soon after training lack anesthesia-resistant memory (Folkers et al. 1993), demonstrating that the locus encodes a key molecular component of the ARM pathway. Understanding the function of the Radish protein and the signaling pathway it regulates is critical to dissecting the molecular underpinnings of this phase of memory.

To characterize the Radish-dependent ARM pathway and its link to cAMP signaling, we compared synaptic connectivity in $r u t^{1}, d n c^{1}, a m n^{1}$, and $r s h^{1}$ mutants. Like rut ${ }^{1}, d n c^{1}$, and $a m n^{1}$, $r s h^{1}$ mutants displayed altered synaptic connectivity, suggesting a link between anesthesia-resistant memory storage and synaptic rewiring. We also show that Radish is a PKA target in vitro and localizes to the cytoplasm and nucleus. Genome-wide microarray analysis identified several altered transcriptional targets that are both shared and divergent in the STM and ARM mutants. Approximately 375 genes implicated in membrane excitability, synaptic transmission, cytoskeletal regulation, cell adhesion, and cellular signaling were differentially regulated in the mutants. We characterized one of these transcriptional targets, Lapsyn (CG15658), in greater detail. Lapsyn encodes a neuronal cell adhesion leucine-rich repeat (LRR) protein that localizes to synapses and regulates synaptic connectivity, suggesting a potential role in learning-induced synaptic modification.

\section{Results}

\section{Altered synaptic morphology in learning and memory mutants}

Genetic analysis of learning and memory mutants in Drosophila has identified distinct components of learning: STM, middle-term memory (MTM), ARM, and LLTM (Fig. 1A). dnc and rut are involved in the formation of STM, which consists of memory during the first $30 \mathrm{~min}$ after training, whereas the amn neuropeptide is involved in the formation of MTM, which occurs between $30 \mathrm{~min}$ and $3 \mathrm{~h}$ after training. $r s \mathrm{~h}$ is required for ARM, and the CREB pathway mediates LLTM. Understanding how these mutants alter the function and structure of synapses may provide insight into the mechanisms of memory consolidation. To evaluate neuronal structure in these learning and memory mutants, we analyzed synaptic connectivity patterns at the well-characterized 3rd instar larval NMJ in $r u t^{1}, d n c^{1}$, and $a m n^{1}$ mutants and compared them with Canton S (CS) controls (Fig. 1B-C). Previous studies had suggested defects in synaptic morphology in $d n c^{1}$ and rut $^{1}$ (Zhong and Wu 1991; Renger et al. 2000). We quantified axonal branching, innervation length along the muscle and synaptic varicosity number at NMJs at muscles 6 and 7 by performing immunocytochemistry on wandering 3rd instar larvae with anti-Synaptotagmin I or anti-Complexin antibodies. All of the mutants were found to alter connectivity patterns compared with controls. rut ${ }^{1}$ mutants showed a $28 \%$ decrease in synaptic varicosity number when normalized to muscle surface area $(P<$ 0.005 , Student's $t$-test). In addition, they displayed a $29 \%$ decrease in axonal branch number $(P<0.001)$ and a $20 \%$ decrease in innervation length $(P<0.01)$. $d n c^{1}$ mutants displayed a $33 \%$ increase in varicosity number normalized to muscle surface area $(P<0.01)$. Our findings in $d n c^{1}$ and $r u t^{1}$ are similar to previous observations (Zhong and $\mathrm{Wu}$ 1991; Renger et al. 2000). The $a m n^{1}$ mutant showed a $19 \%$ decrease in axonal branch number compared with controls $(P<0.05)$ without affecting synaptic varicosity number or innervation length. These results suggest that learning and memory mutants disrupting STM and MTM have altered synaptic connectivity patterns at larval NMJs.

\section{Radish regulates synaptic connectivity and encodes a PKA substrate that localizes to the cytoplasm and nucleus}

The Radish protein is encoded by CG15720 and contains 23 predicted PKA phosphorylation sites and five bipartite nuclear localization signals (NLSs), which overlap with several of the PKA sites (Folkers et al. 2006). How the Radish protein contributes to ARM is unknown. The prominent role of PKA in Drosophila learning and memory suggests that PKA activation during the initial phases of memory may directly impinge upon Radish function, providing a transition from short- to long-term memory storage mechanisms, similar to the PKA-CREB link in LLTM. Based on the predicted PKA sites contained in the Radish protein, we assayed whether Radish was as a PKA target in vitro. Full-length Radish proved difficult to purify as a recombinant protein. To overcome these difficulties in protein stability, we generated a recombinant GST-Radish fusion protein from a 102-residue fragment (Ala 383 to Pro 484) predicted to be surface exposed and containing six predicted PKA sites. We assayed phosphorylation of this fragment in in vitro assays with recombinant PKA. GST-Radish underwent PKA-dependent phosphorylation, whereas GST alone was not phosphorylated by PKA (Fig. 2A), suggesting that Radish function may be regulated through the CAMP-PKA signaling pathway.

Given that $r u t^{1}, d n c^{1}$, and $a m n^{1}$ mutants alter synaptic connectivity at the larval NMJ, we assayed whether axonal branching and synapse formation might be altered in $r s h^{1}$ mutants as well (Fig. 2B-D). Compared with CS, $r s h^{1}$ mutants displayed a $21 \%$ increase in varicosity number $(P<0.001)$ (Fig. $2 \mathrm{~B})$ and a $15 \%$ decrease in innervation length $(P<0.05)$ (Fig. 2C), resulting in a compact innervation pattern at the NMJ. These results indicate that $r s h^{1}$ mutants have altered axonal branching and synaptic growth.

To analyze the cellular compartment in which the Radish protein may function to regulate ARM and synaptic connectivity, we generated UAS-rsh-GFP transgenes and evaluated Radish localization in transgenic animals. Previous analysis of Radish immunoreactivity revealed neuropil staining in the calyx, peduncle, and lobes of the mushroom bodies (Folkers et al. 2006), suggesting localization to synapses. The presence of multiple NLSs within the protein suggests Radish might also act in the nucleus. Transgenic animals expressing UAS-rsh-GFP in larval salivary glands showed prominent nuclear accumulation of Radish-GFP, overlapping with DAPI staining (Fig. 2E,F). Weaker staining in the cytoplasm of salivary gland cells was also observed (Fig. 2E). Similarly, we observed robust nuclear accumulation of Radish-GFP when driven with the muscle driver mef2-GAL4 (Fig. 2G). When the pan-neuronal elav ${ }^{\text {C155-GAL4 }}$ driver was used to express UAS-rshGFP in the brain, Radish was observed in the cytoplasm of neuronal cell bodies and in larval axons (Fig. 2H). Staining in presynaptic terminals was not readily apparent. These data suggest that Radish can localize to both the cytoplasm and nucleus depending on cell type. Given the weak homology of Radish to arginine/serine-rich RNA-splicing factors and the overlap of several NLS and PKA phosphorylation sites, an attractive hypothesis is that the protein might shuttle in and out of the nucleus in a PKA-dependent manner to regulate mRNA splicing, stability, or 
A
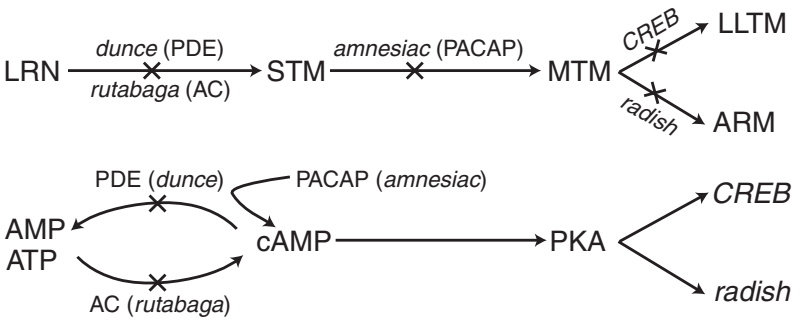

B
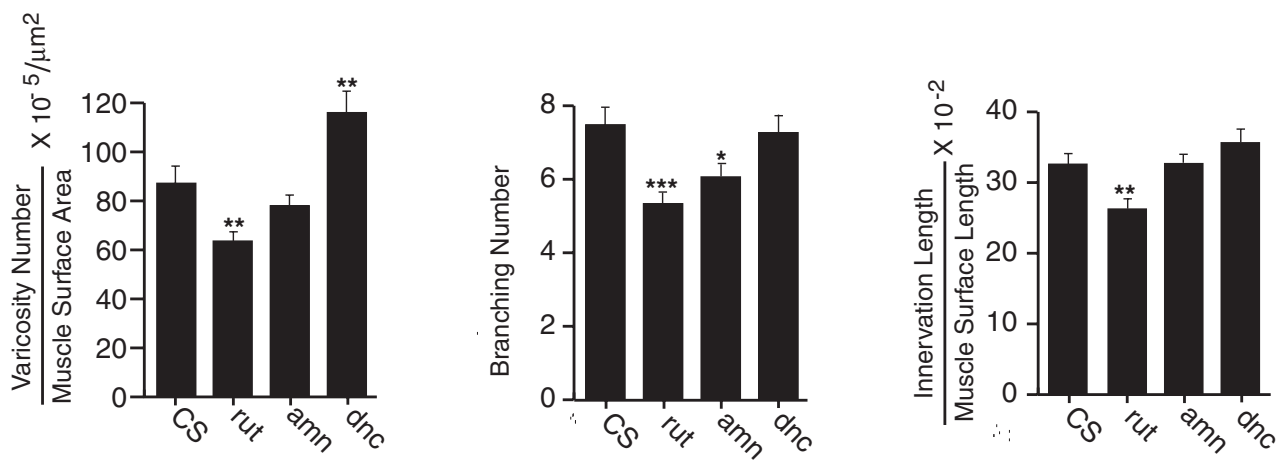

C
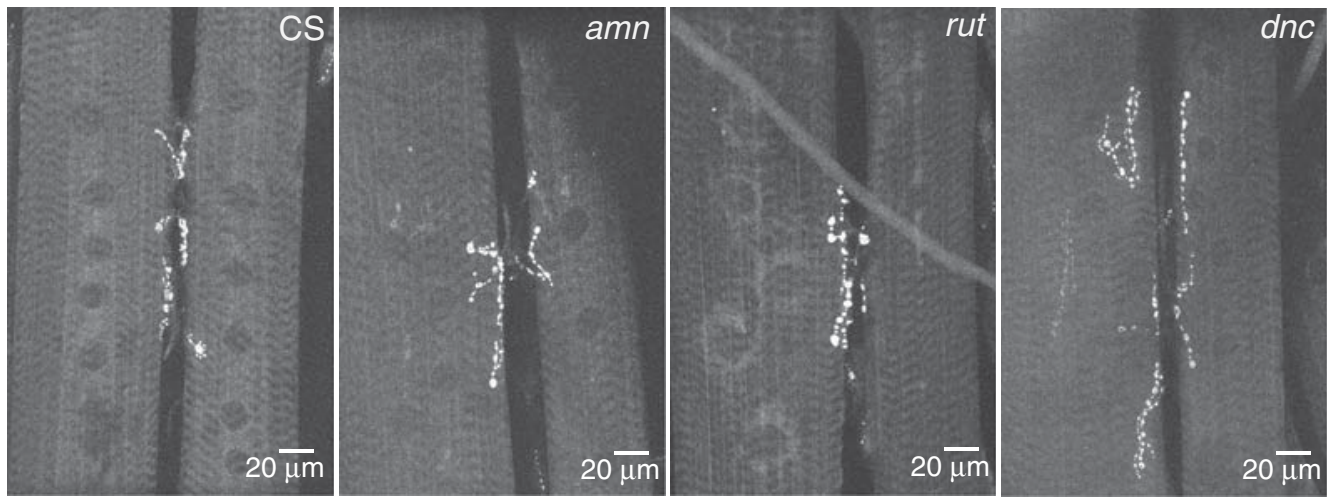

Figure 1. Drosophila learning and memory mutants have altered synaptic connectivity. $(A)$ Diagram of characterized memory phases (top) and their underlying molecular pathways (bottom). (B) Quantification of NMJ morphology changes at muscles 6/7 in segments A3-A5 in 3rd instar larvae. Average varicosity number normalized to muscle surface area was decreased in rut ${ }^{\prime}(63.6 \pm 7.1)$ and increased in $d n c^{\prime}(116 \pm 8.0)$ compared with CS controls $(87.2 \pm 7.1)$. Average nerve branch number was reduced in $r^{1}{ }^{1}(5.3 \pm 0.7)$ and $a m n^{1}(6.1 \pm 0.8)$ compared with CS (7.5 \pm 0.5$)$. Average innervation length normalized to muscle surface length was decreased in rut ${ }^{1}(0.26 \pm 0.013)$ compared with CS $(0.33 \pm 0.017)$. The numbers of NMJs analyzed were: CS (27), rut ${ }^{1}$ (42), $d n c^{1}$ (48), amn $n^{\top}$ (30). P-values for Student's $t$-test: $\left.\left.\left(^{*}\right) P<0.05 ;{ }^{* *}\right) P<0.01 ;{ }^{* * *}\right) P<0.001$. Error bars are SEM. (C) Representative muscles 6/7 NMJs in segment A3 immunostained with anti-Syt 1 antiserum for the indicated genotypes.

localization. Future studies will be required to determine whether and how PKA alters the distribution of Radish within neurons.

\section{Microarray analysis shows altered transcription in Drosophila learning and memory mutants}

Based on the synaptic connectivity defects at larval NMJs observed in $d n c^{1}, r u t^{1}, a m n^{1}$, and $r s h^{1}$ mutants, we were interested in exploring how altered transcription might contribute to these phenotypes. To identify transcriptional changes in the brain, we used Affymetrix DNA microarray chips to detect differences in gene expression in the mutants compared with CS controls using triplicate chips for each genotype (Fig. 3A). We isolated total RNA from 600 pooled heads for each chip to enrich for neuronal transcripts and reduce individual variability in gene expression. RNA was extracted from 3-4 d old males reared at $22^{\circ} \mathrm{C}$ and sacrificed at the same time of day (2-4 PM) to reduce circadian gene expression changes. The Affymetrix Drosophila chip covers most of the $\sim 14,000$ ORFs in the genome. We analyzed transcripts whose mRNA signal strength was called as present $(\mathrm{P})$ or marginal $(\mathrm{M})$ on at least one of the three chips. Using this criterion, we detected $\sim 50 \%$ of known Drosophila genes in adult male heads (Supplemental Table 1). We probed for expression differences between chips for each mutant using statistical algorithms included in the Affymetrix Microarray Suite MAS 5.0 software, as well as Student's $t$-test and the Mann-Whitney $U$-test. For each differentially expressed transcript, we required a $P$-value $<0.05$, a $50 \%$ or greater change in gene expression between strains, and signal strength called as present (P) or marginal (M) on at least one chip (Supplemental Table 2).

To functionally categorize the differentially expressed genes, we performed DAVID (The Database for Annotation, Visualization, and Integrated Discovery) analysis with the gene ID conversion tool. Each altered gene was subsequently checked by BLAST analysis to identify homologs and previously characterized structural domains. The genes were categorized according to known or 
A

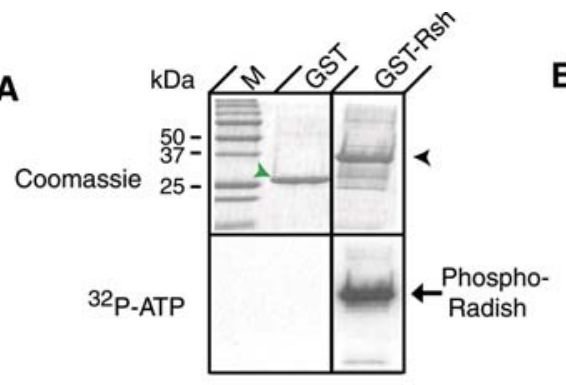

B

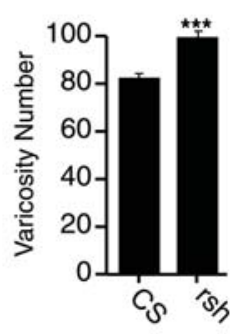

C $\stackrel{0}{\circ}$

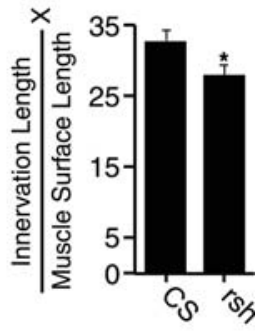

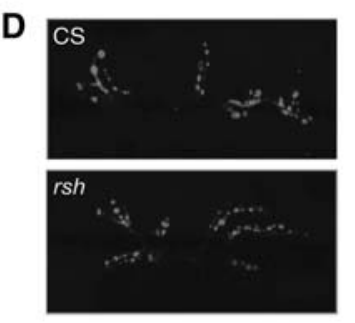

E
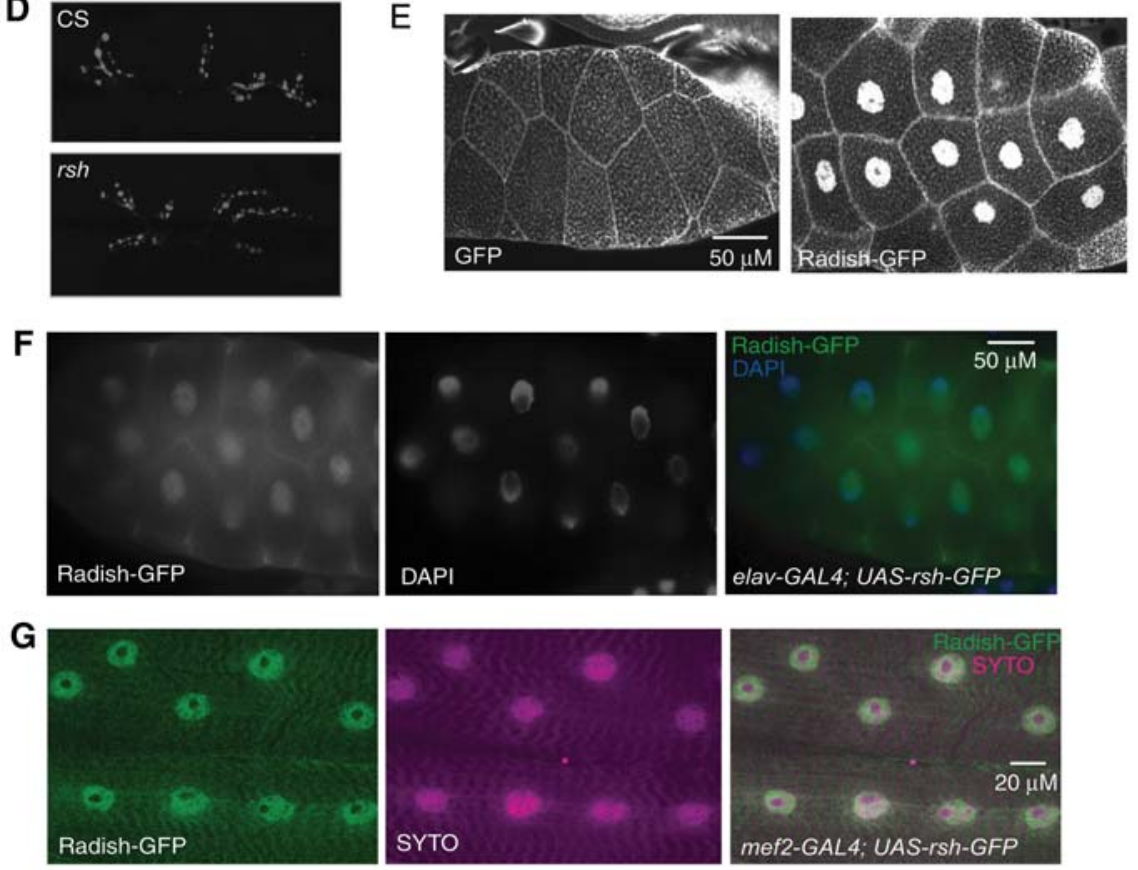

H
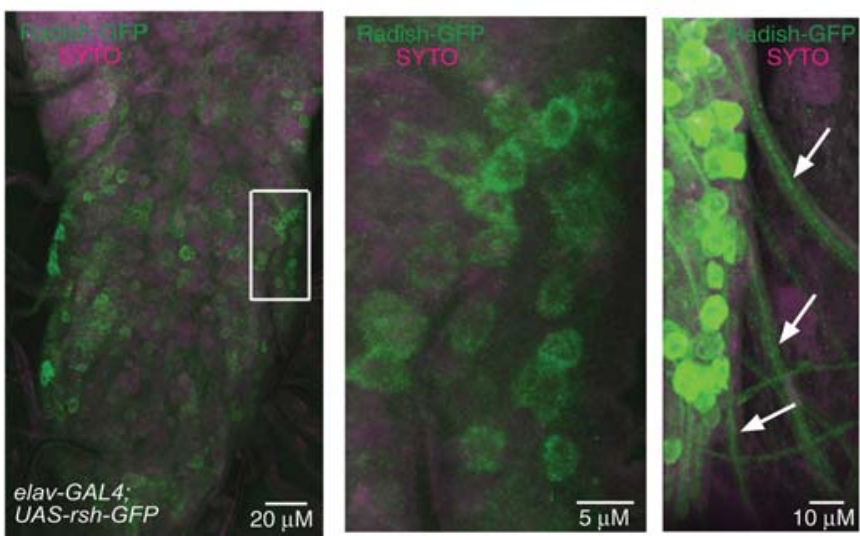

Figure 2. Characterization of Radish localization and function. (A) Phosphorylation of a Radish protein fragment (amino acids 383-484) by the catalytic subunit of PKA. Ten micrograms of recombinant GST alone (green arrowhead) or GST-Radish (black arrowhead) was incubated with $0.5 \mu \mathrm{g}$ of the PKA catalytic subunit in the presence of $\left[\gamma^{32}\right.$ P]ATP. Coomassie staining of the SDS-PAGE gel is shown at top, and the bottom panel shows the autoradiograph. (B) Quantification of average varicosity number at muscles 6/7 NMJs in segment A3 in 3rd instar CS or rsh ${ }^{1}$. Average varicosity number was significantly increased in $r h^{1}(99.2 \pm 3.2, n=14)$ compared with CS $(82.2 \pm 2.1, n=11)$. $\left.{ }^{* * *}\right) P$-value of Student's $t$-test $(P<0.001)$. Error bars represent SEM. (C) Quantification of average innervation length normalized to muscle surface length at muscles 6/7 NMJs in segments A3-A5 in 3rd instar CS or $r s h^{1}$ larvae. Innervation length was significantly decreased in $r h^{1}$ mutants $(0.27 \pm 0.014, n=39)$ compared with CS $(0.36 \pm 0.017, n=27)$. (*) $P$-value of Student's $t$-test $(P<0.05)$. Error bars represent SEM. $(D)$ Immunocytochemistry with anti-Complexin antiserum on muscles $6 / 7$ NMJs in segment A3 in 3rd instar larvae. (E,F) Expression of UAS-rsh-GFP by elav ${ }^{C 155}$-GAL4 in 3rd instar larvae. (E) Radish-GFP was concentrated in the nucleus of salivary gland cells compared with GFP alone. $(F)$ Colocalization of Radish-GFP and DAPI in the nucleus of salivary gland cells. $(G)$ RadishGFP was concentrated in the nucleus of muscle cells when driven by the Mef2-GAL4 driver. Nuclei were counterstained with SYTO orange (shown in magenta). (H) Radish-GFP localized to neuronal cell bodies in the ventral nerve cord and axons (arrows) in 3rd instar larvae. (Middle) A magnified view of the framed area in the left panel. (Right) Radish-GFP in nerve bundles (arrows) exiting the ventral nerve cord. Nuclei were counterstained with SYTO orange (shown in magenta). 


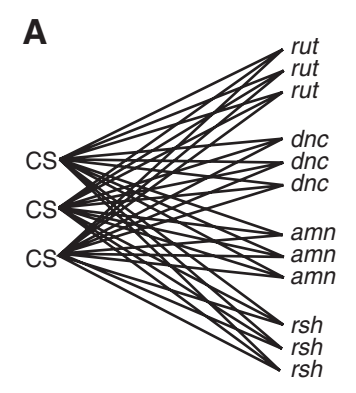

C

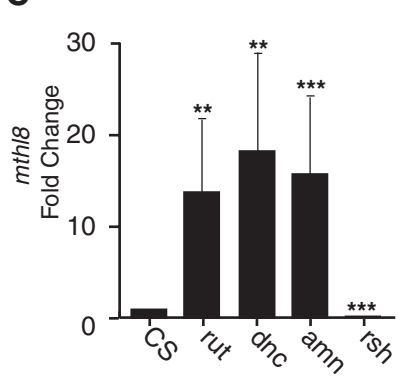

B

\begin{tabular}{lcccc} 
Functional Category & $\begin{array}{c}\text { rut } \\
(158)\end{array}$ & $\begin{array}{c}d n c \\
(89)\end{array}$ & $\begin{array}{c}a m n \\
(70)\end{array}$ & $\begin{array}{c}r s h \\
(153)\end{array}$ \\
\hline Membrane Excitability & 5 & 2 & 4 & 6 \\
Synaptic Transmission & 5 & 3 & 1 & 1 \\
Cell Adhesion & 2 & 3 & 1 & 5 \\
Cytoskeletal Regulation & 5 & 3 & 4 & 6 \\
Cell Signaling & 37 & 34 & 21 & 44 \\
Others & 104 & 44 & 39 & 91 \\
\hline
\end{tabular}
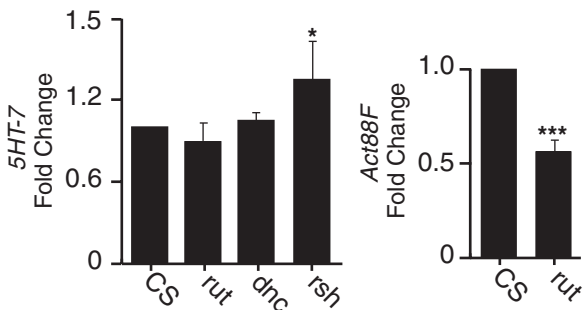
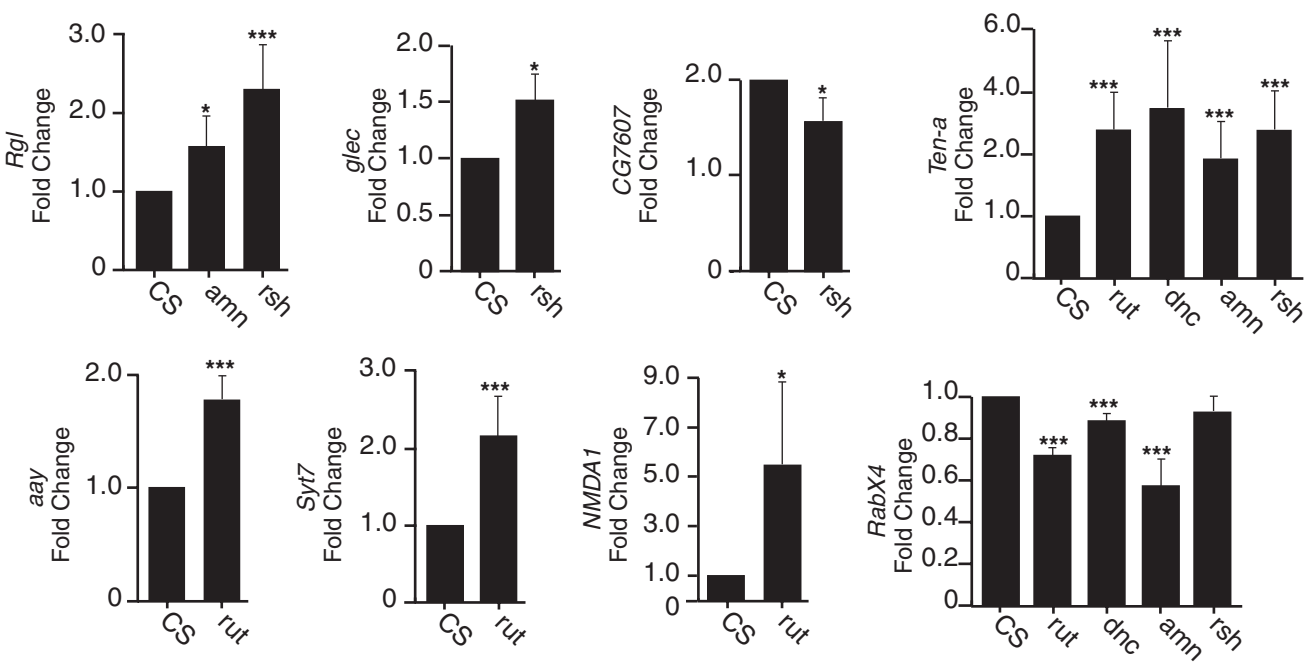

D

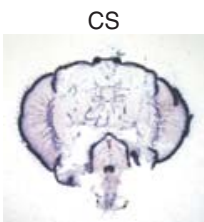

mthl8

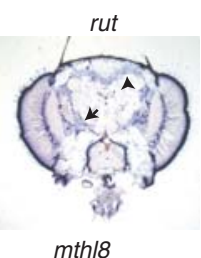

mth/8

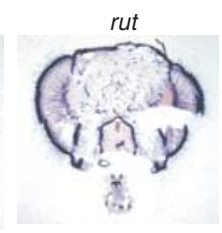

mth/8-Sense

Figure 3. Microarray analysis of learning and memory mutants. ( $A$ ) Comparison matrix used for the microarray analysis. ( $B$ ) Distribution of transcriptionally altered genes identified by genome-wide microarray screens according to the functional category for each genotype. ( $C$ ) Summary graphs of real-time quantitative RT-PCR results for a subset of the altered gene products. $P$-values for Student's $t$-test: $(*) P<0.05 ;(* *) P<0.01 ;(* * *) P<$ 0.001. Error bars represent SEM. Four trials were run for each indicated genotype. (D) In situ hybridization on adult CS and rut ${ }^{1}$ brain sections with mth/8 cRNA probes. mth/8 was up-regulated in neuronal cell bodies (arrowhead) and in visual system neurons (arrow) in rut ${ }^{1}$ compared with CS. A sense strand $m$ th/8 CRNA probe was used as a negative control.

putative functions based on sequence similarity as indicated in Supplemental Table 2. The number of altered genes in individual functional classes for each genotype is shown in Figure 3B. We identified more differentially expressed genes in $r u t^{1}(158)$ and $r s h^{1}$ (153) mutants than in $d n c^{1}$ (89) and $a m n^{1}$ (70) mutants. Gene products with predicted roles in membrane excitability in $r u t^{1}(2.18 \%)$ and $a m n^{1}(1.72 \%)$, and in cell adhesion in $d n c^{1}$
(2.4\%) and $r s h^{1}(4 \%)$, were more likely to be transcriptionally regulated compared with other functional categories (Fig. 3B; Supplemental Fig. 1). We focused on the analysis and verification of altered genes predicted to be involved in membrane excitability, synaptic transmission, cytoskeletal regulation, cell adhesion, and cell signaling, as these pathways have been linked to synaptic plasticity. 
Altered transcription detected in rutabaga mutants

Among the genes abnormally expressed in $r u t^{1}$ was the catalytic subunit of PKA (Pka-C1, also known as DCO), which was up-regulated 1.5-fold. Mutations in PKA lead to learning and memory deficits (Skoulakis et al. 1993), whereas up-regulation of PKA function modulates synaptic growth (Yoshihara et al. 2005). Adh transcription factor 1 (Adf1), which encodes a Myb-related transcription factor, was also up-regulated in rut ${ }^{1}$. Notably, Nalyot ( nal $^{P 1}$ ), a hypomorphic allele of Adf1, shows mild deficits in STM, severe defects in LTM, and reduced synaptic growth (DeZazzo et al. 2000). Other interesting signaling components altered in $r u t^{1}$ included GTPase-activating protein 1 (Gap1), a negative regulator of Ras in RTK signaling (Gaul et al. 1992); Dorsal ( $d l)$, encoding an NF-к $\beta$ transcription factor that regulates mushroom body formation (Nicolai et al. 2003) and synaptic growth (Cantera et al. 1999); Med11, an RNA polymerase II transcriptional regulator that controls dendritic branching (Parrish et al. 2006); and $\operatorname{cln} 3$, a Drosophila homolog of the human $\operatorname{cln} 3$ gene mutations that cause the neurodegenerative disorder juvenile neuronal ceroid lipofuscinosis.

Beyond signaling components, we detected altered transcription of several other gene classes in rut ${ }^{1}$ mutants. Among the transcriptionally altered genes regulating membrane excitability and synaptic communication were Nervana 2 (nrv2), a $\beta$ subunit of the sodium/potassium ATPase; NMDA1, which encodes the Type 1 Drosophila NMDA receptor homolog; Syt 7, a high-affinity calcium sensor of the synaptotagmin family; lap, the AP-180 homolog that regulates clathrin-mediated endocytosis; $\alpha$-SNAP, a soluble N-ethylmaleimide-sensitive (NSF) attachment protein; CG3822, a predicted kainite-selective glutamate receptor; $5-H T 7$, a serotonin receptor; and ebony $(e)$, which regulates dopamine formation. Gene products implicated in cytoskeletal dynamics were also altered, including diaphanous (dia), a regulator of actin polymerization that modulates synaptic growth (Pawson et al. 2008); Ciboulout (cib), which participates in actin filament assembly and brain development; and CG6480, the Drosophila homolog of human facioscapulohumeral muscular dystrophy region gene 1 ( $f r g-1$ ). Loci with predicted functions in cell adhesion were also misregulated, including the leucine-rich repeat (LRR) domain transmembrane proteins, CG15658 and CG18249. Finally, several components of the RNA-induced silencing complex (RISC) were altered, including Vasa intronic gene (vig) and argonaute 2 (ago2).

\section{Altered transcription detected in dunce mutants}

Given that $d n c^{1}$ and $r u t^{1}$ have opposite effects on cAMP levels, we expected to find distinct transcriptional changes, along with some conserved changes. Only a few transcripts were altered in both rut $^{1}$ and $d n c^{1}$, including 5-HT7, cib, and mthls (a Methuselah-like $G$ protein coupled receptor). Among the unique genes that were changed in $d n c^{1}$ mutants was Tequila, a neurotrypsin serine protease transcriptionally induced by learning stimuli and required for LTM (Didelot et al. 2006). Other transcripts altered in $d n c^{1}$ mutants were tan $(t)$, a $\beta$-alanyl-dopamine hydrolase; Punch (Pu), a GTP cyclohydrolase; CG4587, an $\alpha_{2} \delta$ calcium channel subunit; neurexin IV (Nrx-IV), a glial-enriched cell surface protein; Ten- $a$, an EGF-containing transmembrane protein enriched on developing axons; and hairless $(H)$, a regulator of Notch signaling (Supplemental Table 2).

\section{Altered transcription in amnesiac mutants}

$a m n^{1}$ mutants showed the least altered transcriptional profile of the mutants evaluated. Among the up-regulated transcripts we detected in $a m n^{1}$ was Frequenin 2 ( frq2), a neuronal calcium sensor
1 (NCS-1) homolog shown to regulate synaptic connectivity and synaptic transmission (Dason et al. 2009). Calcium/calmodulindependent protein kinase (Caki, also called CamguK or CASK) was also up-regulated in $a m n^{1}$. Caki acts as a gain control for a CaMKII-dependent molecular switch during activity-dependent plasticity in Drosophila (Lu et al. 2003; Hodge et al. 2006). Rpd3, a histone deacetylase homolog of mammalian HDAC1, was downregulated in $a m n^{1}$. Several genes controlling membrane excitability and neuronal signaling were also altered, including $G A B A-B$ receptor subtype 3 (GABA-B-R3); Shaking B (shakB), a Drosophila innexin required for gap junction formation; Tomosyn, a regulator of synaptic vesicle fusion; Split ends (spen), a transcriptional corepressor that regulates neuronal development; Ral guanine nucleotide exchange factor $(\mathrm{Rgl})$, a RalGEF homolog implicated in synaptogenesis (Liebl et al. 2006); and roughoid (ru, also termed rhomboid 3), a serine-type peptidase involved in the EGF pathway.

\section{Altered transcripts observed in radish mutants}

Given that $r s h$ is the least characterized regulator of memory formation at the molecular level among the mutants we analyzed, we were interested to see whether $r s h^{1}$ had a particular transcriptional profile that would distinguish it from the STM and MTM mutants. Genes that were altered in both $r s h^{1}$ and the other learning mutants were Caki, AGO2, nrv2, dia, dl, CG15658, Lk6, CG7607, mthl8, and Rgl. Among the genes uniquely altered in $r \mathrm{sh}^{1}$ was the up-regulated transcript rugose $(\mathrm{rg})$, which encodes a PKA anchor protein (DAKAP550) required for later stages of memory formation in Drosophila (Schwaerzel et al. 2007). Adenyly cyclase 35C (Ac13E), another component of the cAMP pathway, was also up-regulated in $r s h^{1}$. In addition to these loci, a number of genes involved in synaptic transmission and membrane excitability were altered. The Shaker (Sh) voltage-dependent potassium channel encoding $\mathrm{I}_{\mathrm{A}}$ was up-regulated in $r s h^{1}$. Shaker has wellestablished roles in the regulation of neurotransmitter release and synaptic growth (Jan et al. 1977; Ganetzky and Wu 1982; Budnik et al. 1990), and Sh mutants display deficits in olfactory learning (Cowan and Siegel 1986; Dubnau and Tully 1998). Open rectifier $K+$ channel 1 (Ork1), a potassium-selective leak channel (Goldstein et al. 1996), and Rim, a Rab3-interacting active zone protein previously implicated in LTP (Castillo et al. 2002), were also altered in $r s h^{1}$. We identified transcriptional changes in several genes involved in cytoskeletal regulation or cell adhesion, including Disabled (Dab), which encodes a protein that colocalizes with Ableson kinase (Abl) in axons (Gertler et al. 1993); Grip163, a $\gamma$-tubulin ring complex ( $\gamma$ TuRC) subunit involved in microtubule nucleation (Gunawardane et al. 2000); and capping protein $\beta(c p b)$, an F-actin capping protein subunit (Delalle et al. 2005). In addition, we detected changes in transcripts for spire (spir), an actin nucleation protein; sticks and stones (sns), a cell-adhesion protein with eight Ig domains and a fibronectin type III domain; gliolectin (glec), a carbohydrate-binding protein found in glia; and beat- $V b$, an Ig domain-containing protein expressed in the CNS.

A number of loci regulating cell signaling were also altered in $r s h^{1}$. These transcripts included CG11399, the Drosophila ortholog of human phosphorylated carboxy-terminal domain (CTD) interacting factor 1 (PCIF1). The PCIF1 protein regulates mRNA synthesis through interactions between its WW domain and hyperphosphorylated RNA polymerase II (Fan et al. 2003). Overexpression of CG11399 induces a Notch gain-of-function phenotype (Bejarano et al. 2008), suggesting that CG11399 may modulate the transcription of mRNAs involved in Notch signaling. SH3PX1, which encodes an SH3-PHOX domain-containing protein (Kurten et al. 1996; Howard et al. 1999; Worby et al. 2001) that is a component of the DSCAM (Down syndrome cell 
adhesion molecule) complex (Clemens et al. 2000; Worby et al. 2001), was also up-regulated. Drosophila SH3PX1 can interact directly with WASp (Worby et al. 2001), suggesting that it may link DSCAM signaling to WASp regulation of the actin cytoskeleton. Protein tyrosine phosphatase $61 F$ (ptp61F), an ortholog of mammalian PTBIB, a nontransmembrane tyrosine phosphatase, was down-regulated by 4.7 -fold. Ptp61F/PTB1B is expressed in the Drosophila CNS and acts as a negative regulator of JAK/STAK signaling (Baeg et al. 2005). It also functions with Ableson kinase interacting protein (Abi) to regulate lamellipodial structure through the SCAR/WAVE complex (Huang et al. 2007). Also down-regulated were protein phosphatase $2 A$ at 29B (Pp2A-29B), Elongator protein 3 (ELP3), which has been implicated in RNA processing and neurodegeneration (Simpson et al. 2009), and Protein Phosphatase 2C (CG17746), a regulator of JNK signaling. Future studies will be needed to assess how the altered transcriptional profiles found in the learning and memory mutants contribute to their underlying behavioral and synaptic defects.

\section{Verification of a subset of the transcriptional targets}

To begin verifying the transcriptional profiles obtained by microarray analysis, we analyzed a subset of the identified genes using real-time quantitative RT-PCR and semi-quantitative RT-PCR (Fig. 3C). We chose 15 transcripts for quantitative PCR verification, including both up-regulated and down-regulated gene products. Of the 15 transcripts assayed, 12 showed similar changes by quantitative RT-PCR as reported by the microarrays (Fig. 3C; Supplemental Fig. 2). Three transcripts, including 5-HT7 (Fig. 3C; Supplemental Fig. 2), CG3775, and CG6852, showed no change, indicating an $\sim 80 \%$ concordance rate between our quantitative RT-PCR and microarray results, based on this smaller set.

We previously performed an extensive microarray analysis of activity-regulated transcripts using temperature-sensitive Drosophila hyper- and hypo-excitability mutants and control animals treated with epileptic drugs (Guan et al. 2005). In that analysis, a few transcripts were identified that displayed bidirectional modification by increased or decreased activity. One such transcript was $m$ thls, a Methuselah-like G-protein coupled receptor, which we found to be up-regulated in $r u t^{1}, d n c^{1}$, and $a m n^{1}$ mutants (Supplemental Table 2). We analyzed the up-regulation of $m$ thl 8 by performing in situ expression analysis on adult head sections and compared expression patterns between rut ${ }^{1}$ and controls. $m$ thl 8 was present at low levels throughout the nervous system in CS brains, but was broadly up-regulated in rut ${ }^{1}$ brains (Fig. 3D), indicating $m$ thls transcription is directionally controlled by the levels of neuronal activity and is up-regulated in several learning and memory mutants.

\section{Characterization of the Lapsyn cell adhesion protein}

Our microarray analysis identified $\sim 375$ altered genes, encompassing several functional classes. Only $8 \%$ (30 genes) of the loci identified in this study overlapped with our previous analysis of activity-related genes in the Drosophila brain (Guan et al. 2005; Supplemental Table 2). Given the role of enhanced neuronal activity in driving synaptic plasticity, this subgroup of overlapping genes was of particular interest. Among the genes found to be transcriptionally altered in both learning and memory mutants and by changes in neuronal activity were the soluble growth factor $l d g f 1$, the FGF receptor ligand $B n l$, the PKC homolog inaC, AGO2 of the RNAi silencing complex, the Lk6 serine/threonine kinase, the vesicle fusion regulator $a-S N A P$, and the cell adhesion proteins CG15658 and CG7607. In view of the connection between increased neuronal activity, synaptic plasticity, and altered neuronal connectivity, we were interested in further characterizing gene products that might contribute to the synaptic structural changes observed in the learning and memory mutants evaluated. We focused on the CG15658 locus, which encodes a cell adhesion molecule with four leucine-rich repeat (LRR) extracellular domains (Fig. 4A). We termed the CG15658 protein Lapsyn (Leucine-rich repeat activity-regulated protein at synapses) to reflect its activity regulation and structural motifs. Lapsyn has not been previously characterized and has no known function. Lapsyn RNA levels were altered both in activity-dependent seizure mutants and in the learning and memory mutants rut ${ }^{1}$ and $r s h^{1}$, prompting us to test whether it might regulate synaptic connectivity.

Lapsyn displays homology to several mammalian proteins in its extracellular domain, with $29 \%$ identity and $52 \%$ similarity to the netrin-G ligand (NGL) family in mammals. Unlike its conserved extracellular LRR domains, the short intracellular C terminus of Lapsyn lacks homology to known proteins. To confirm the changes in mRNA levels observed by microarray analysis (Lapsyn was up-regulated in the hyperexcitability temperature-sensitive mutants $\operatorname{set}^{t s 1}(2.1, P<0.001)$, slo ${ }^{t s 1}(2.7, P<0.01)$, zydeco (2.3, $P<0.05)$, and jive $(2, P<0.001))$, we performed semiquantitative RT-PCR in the activity mutants following a temperature-shock paradigm with a 30 -min recovery period (Fig. 4B) or a 24-hour recovery period (Fig. 4C). Lapsyn was up-regulated in the activity mutants tested under both conditions. We also analyzed Lapsyn expression levels by semiquantitative RT-PCR in the learning and memory mutants and found it to be up-regulated in rut ${ }^{1}$ and $r s h^{1}$, but not in $a m n^{1}$ and $d n c^{1}$ (Figs. 3C, 4D; Supplemental Fig. 2), consistent with the microarray data.

To examine Lapsyn mRNA expression, we performed in situ hybridization on embryos with antisense and control sense probes. Lapsyn was expressed early in development at stage 5 in vitellophages, with expression detected later in the developing midgut and hindgut (Supplemental Fig. 3). mRNA expression in other tissues was low or not detected in CS embryos. We next assayed Lapsyn mRNA expression by in situ hybridization on adult brain sections (Fig. 4E). The endogenous expression level was low in CS animals, although expression was detected throughout the brain rind, where neuronal cell bodies are found in Drosophila. Lapsyn mRNA levels were up-regulated in sei ${ }^{T S 1}$ mutants, with increased expression observed in the medulla region of the visual system and in cell bodies in the dorsal brain rind (Fig. 4C). These data suggest Lapsyn is normally expressed at low levels, but is up-regulated in the nervous system by elevated neuronal activity.

To localize the Lapsyn protein, we generated antiserum against a recombinant N-terminal extracellular sequence of Lapsyn fused to GST. The antiserum did not recognize endogenous protein by Western blot analysis. We next tested whether the antiserum would recognize overexpressed Lapsyn by generating transgenic flies expressing UAS-Lapsyn, allowing transcriptional control using a GAL4 transcriptional activator. Our antiserum detected overexpressed Lapsyn by Western blot analysis (Fig. 4F), but not by tissue immunostaining. As a second approach to evaluate Lapsyn localization, we generated UASLapsyn-GFP transgenic lines. The overexpressed GFP-tagged Lapsyn was detectable by both anti-Lapsyn and anti-GFP antibod-

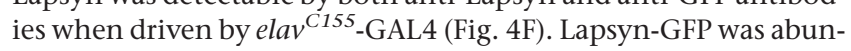
dant in neuronal cell bodies in the brain and ventral nerve cord, and in axons of visual neurons projecting into the laminar plexus of the developing eye disk (Supplemental Fig. 4). At larval NMJs, Lapsyn-GFP accumulated in presynaptic terminals, colocalizing with other presynaptic markers such as the synaptic protein Complexin (Fig. 4G). At higher resolution, Lapsyn-GFP was present in punctate structures present on or near the presynaptic plasma membrane (Fig. 4H,I). We suspected these structures might be presynaptic active zones, which are sites of synaptic 
A
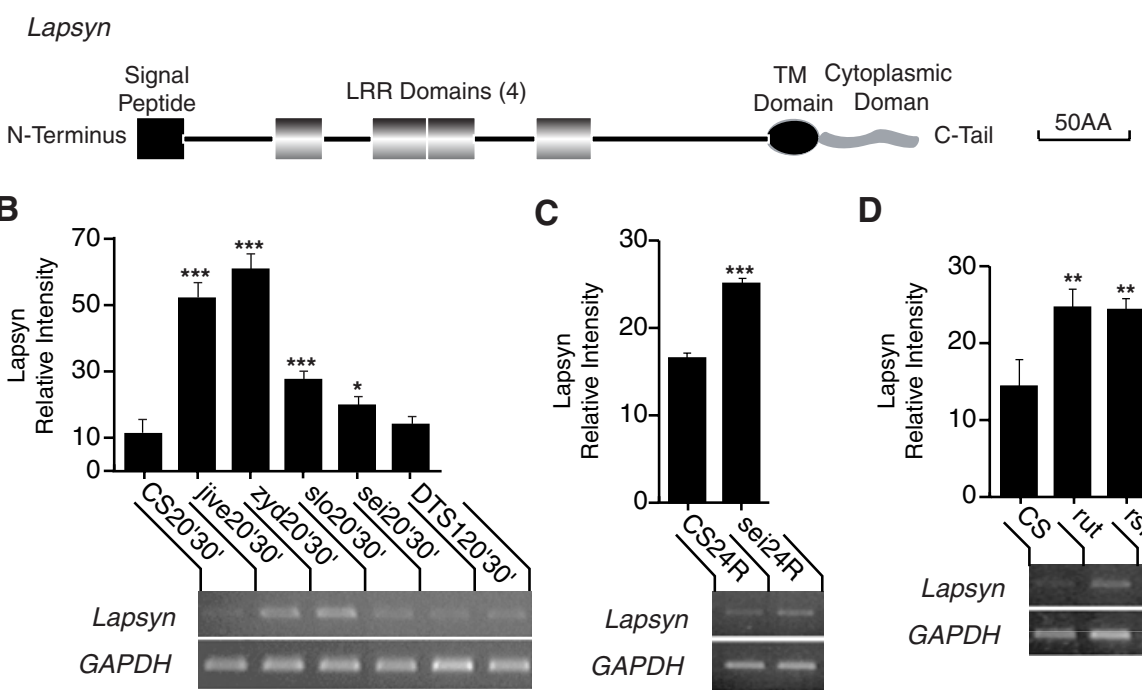

\section{B}

E
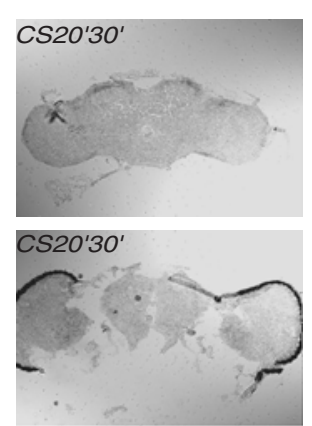

G

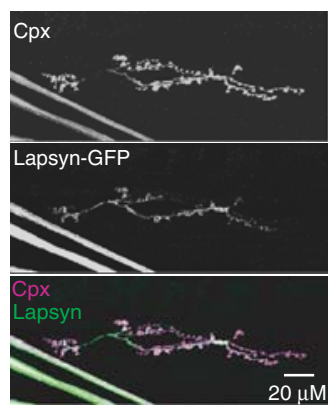

C

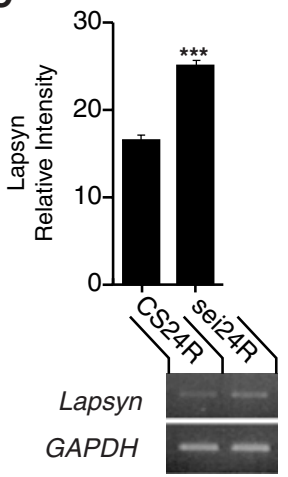

D

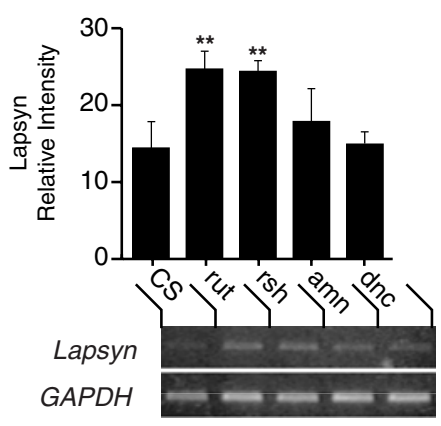

F

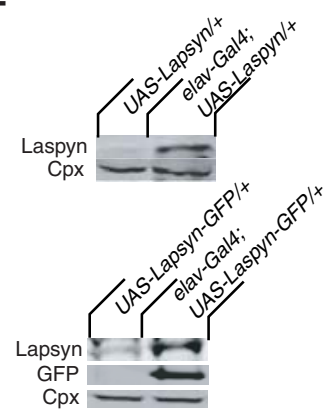

H

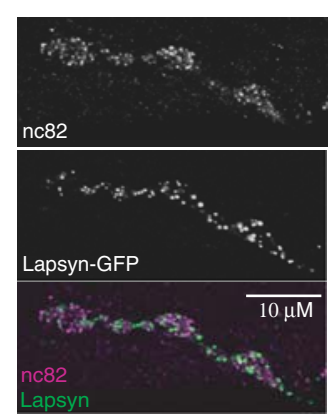

I

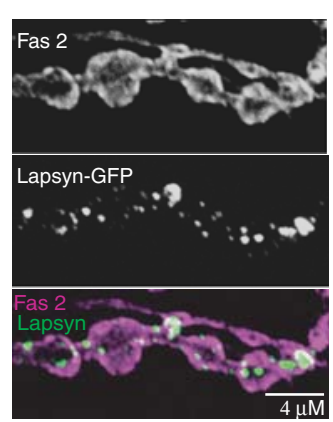

Figure 4. Lapsyn expression and localization at synapses. (A) Domain structure of Lapsyn. (B) Representative gels and quantification of average Lapsyn mRNA expression in temperature-sensitive (TS) seizure mutants compared with CS following a 20-min heat shock and 30 -min recovery period. The following genotypes were tested: CS, $11.3 \pm 4$ relative intensity; ses $B^{\text {jive }}$ ( jive $20^{\prime} 30^{\prime}$ ), $52.1 \pm 4.5,4.6$-fold increase, $P<0.001 ; z y d e c o\left(z y d 20^{\prime} 30^{\prime}\right), 60.9 \pm$

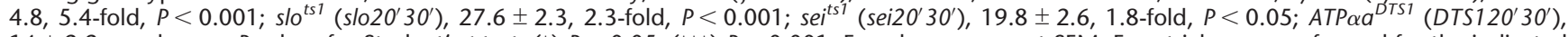
$14 \pm 2.2$, no change. $P$-values for Student's $t$-test: $\left(^{*}\right) P<0.05 ;\left({ }^{* * *}\right) P<0.001$. Error bars represent SEM. Four trials were performed for the indicated genotypes. (C) Representative gel and quantification of average Lapsyn mRNA expression in CS (16.6 \pm 0.6$)$ and seits ${ }^{\text {(25. }} \pm$ $\pm 0.7,1.5$-fold increase, $P<0.001)$ following a 24 -h recovery from a $4 \times 5$-min $38^{\circ} \mathrm{C}$ heat-pulse paradigm. Four trials were performed for the indicated genotypes. $(D)$ Representative gel and quantification of average Lapsyn mRNA expression in learning and memory mutants. Lapsyn was up-regulated in rut ${ }^{1}$ ( $24.7 \pm$ 2.3, 1.7-fold, $P<0.01)$ and $r h^{1}(24.4 \pm 1.3,1.7$-fold, $P<0.01)$, but not in $a m n^{1}(17.9 \pm 4.2)$ or dnc $^{1}$ (14.9 \pm 1.5$)$ compared with CS (14.4 \pm 5.8$)$. Three trials were performed for each genotype. $(E)$ In situ hybridizations to adult head sections. Animals were processed identically and imaged with the same settings for CS (CS 20'30') and seitsi (sei 20'30') following a 20-min heat shock and 30-min recovery period using Lapsyn cRNA probes. Lapsyn expression was increased in neuronal cell bodies (arrowhead) and in the medulla region (arrow) of seits 1 . Four experiments were performed for each genotype. $(F)$ Western blot analysis of transgenic Lapsyn expression driven by elav ${ }^{c 155}$-GAL4 in head extracts of the indicated genotypes. (Top)

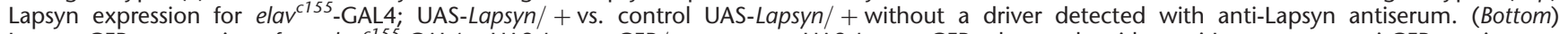
Lapsyn-GFP expression for elav ${ }^{\text {155 }}$-GAL4; UAS-Lapsyn-GFP/+ vs. +; UAS-Lapsyn-GFP detected with anti-Lapsyn or anti-GFP antiserum. Anti-Complexin antiserum was used as a loading control. (G) Representative NMJ from a larva expressing Lapsyn-GFP driven by elav ${ }^{155}$-GAL4 costained for the presynaptic marker Complexin. $(H)$ Representative synaptic boutons from a larva expressing Lapsyn-GFP driven by elav ${ }^{c 155}$-GAL4 costained with the active zone marker anti-nc82. (I) Representative synaptic boutons from a larva expressing Lapsyn-GFP driven by elav ${ }^{c 155}$-GAL4 costained for the periactive zone marker anti-Fas2. 
vesicle docking and release. However, we did not observe colocalization with the active zone marker protein Bruchpilot (antinc82) in double immunostaining experiments (Fig. 4H). We next evaluated colocalization with the periactive zone neuronal cell adhesion protein Fasciclin 2 (Fas2). Periactive zones are regions of the presynaptic terminal enriched for proteins regulating the endocytosis of both synaptic vesicles and activated growth-factor signaling complexes. These regions also contain cell adhesion molecules, such as Fas2, that are known to regulate synaptic growth. Lapsyn-GFP-positive puncta partially overlapped with Fas2 (Fig. 4I), but were often found in spots that extended just beyond Fas2 immunoreactivity. In summary, Lapsyn-GFP is present in both axons and synapses, localizing presynaptically in punctate structures contained within or near periactive zones.

\section{Functional analysis of Lapsyn at synapses}

To analyze the function of Lapsyn in vivo, we generated mutations in the locus by excision of the P-element P\{SUPorP)CG15658[KG09421], which is located 110-bp upstream of the $5^{\prime}$ untranslated region (UTR) of the gene. An imprecise excision $\left(\right.$ Lapsyn $\left.{ }^{z g 1}\right)$ was generated that deleted the 5' UTR and the first two coding exons of the gene (Fig. 5A). The boundaries of the Lapsyn $^{z g 1}$ deletion were confirmed by PCR analysis (Fig. 5B,C). Loss of Lapsyn resulted in embryonic lethality at stage 17 ,

A

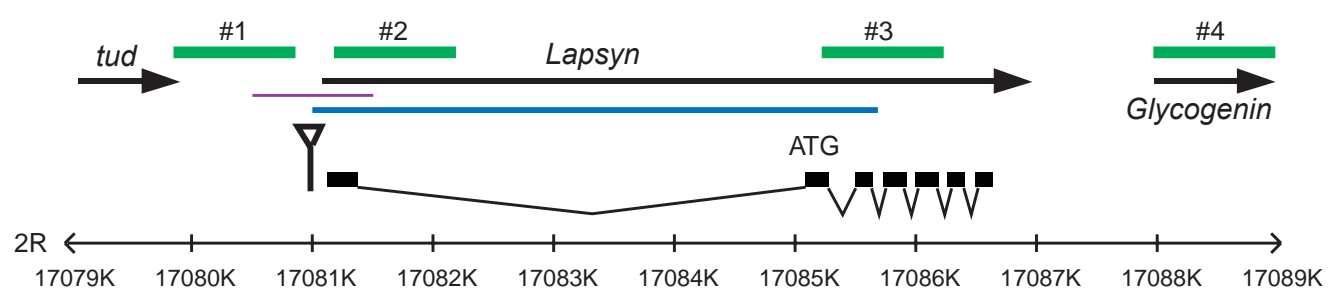

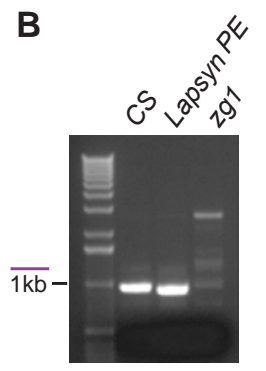

PCR Analysis

C

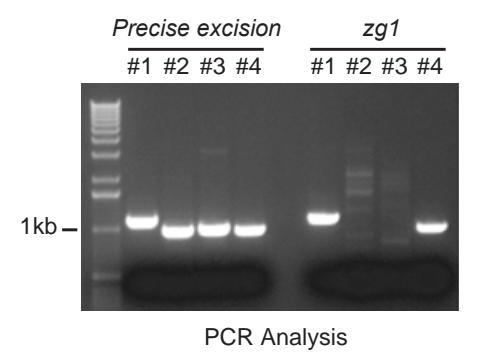

D

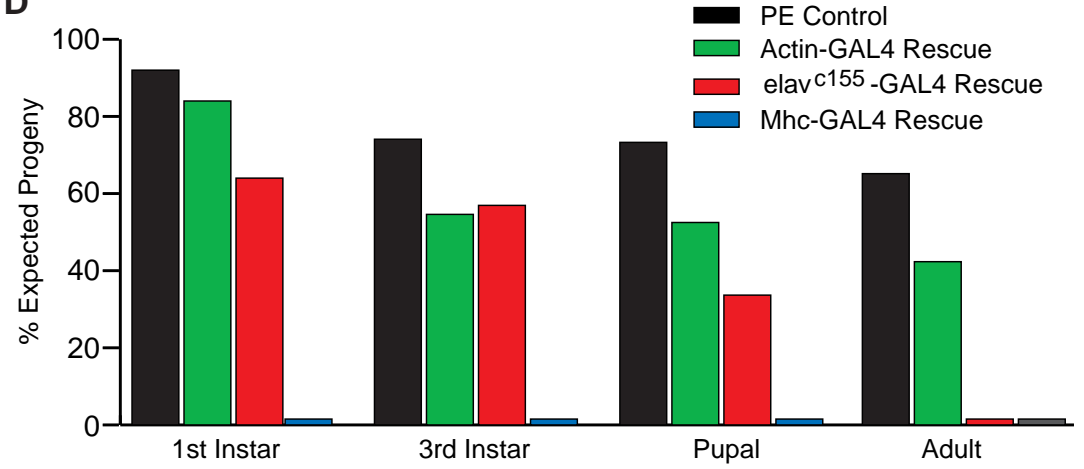

Figure 5. Mutational analysis of Lapsyn. (A) Diagram of the Lapsyn genomic locus with the location of the diagnostic PCR fragments (green/purple bars) used for deletion mapping. A deletion mutant was produced by imprecise excision of a P-element (black triangle) located 110 -bp upstream of the Lapsyn transcription start site. The extent of the deletion, which removes the Lapsyn ATG start site and first two exons, is shown in blue. (B) PCR analysis from genomic DNA spanning the P-element insertion site (purple bar in $A$ ) is shown for CS, a precise excision (Lapsyn ${ }^{P E}$ ) and Lapsyn ${ }^{2 g} 1$. (C) PCR mapping of defined $\sim 1-\mathrm{kb}$ genomic DNA fragments spanning the Lapsyn locus (numbers refer to green bars in $A$ ). The deletion removed the genomic region defined by PCR products \#2 and \#3. (D) Transgenic rescue analysis of Lapsyn ${ }^{2 g}{ }^{1}$ mutants with UAS-Lapsyn driven by actin-GAL4, Mhc-GAL4, and elav ${ }^{c 155}$-GAL4. Crosses were made with zg1/CyO-GFP;UAS-Lapsyn/TM3-GFP males to zg1/CyO-GFP;ACtin-GAL4/TM3-GFP, elav C155-GAL4;Zg1/CyO-GFP, zg1/ CyO-GFP;Mhc-GAL4/TM3-GFP or PE/CYO-GFP;UAS-Lapsyn/TM3-GFP (control) females. A total of 400 embryos were scored from each cross. The percent of expected progeny for each genotype is shown. Expected progeny at each stage for the genotypes tested include: PE control, $92 \% 1$ st instar; 74\% 3rd instar; 73\% pupal; 65\% adult; Actin-GAL4 rescue, 84\% 1st instar; 55\% 3rd instar; 53\% pupal; $42 \%$ adult; elav ${ }^{c 155}$-GAL4 rescue, 64\% 1st instar; $57 \%$ 3rd instar; $34 \%$ pupal; $0 \%$ adult. No rescue of the embryonic lethality was observed in Mhc-GAL4 driven strains. 
indicating that Lapsyn is essential for embryonic development or function. To examine the structure of the nervous system in these mutants, we fixed 0-22-h embryos and stained them with anti-Fas2 antiserum to label axons. Lapsyn ${ }^{z g 1}$ mutants did not show obvious developmental defects in the formation of the CNS or PNS, with normal CNS commissure formation and PNS axonal pathfinding (Supplemental Fig. 5). To determine whether we could potentially rescue the lethality of Lapsyn ${ }^{z g 1}$ mutants, we overexpressed a UAS-Lapsyn transgene ubiquitously using an actin-GAL4 or tubulin-GAL4 driver. Overexpression of UASLapsyn by actin-GAL4 resulted in a partial rescue of Lapsyn ${ }^{z g 1}$ / Lapsyn $^{\text {zg1 }}$ (Fig. 5D) and Lapsyn ${ }^{\text {zg1 }} / D f(2 R)$ Exel6072 (a large deletion that removes Lapsyn, data not shown). Compared with the embryonic lethality of null mutants, $42 \%$ of rescued homozygous mutant animals emerged as adults and showed no obvious behavioral abnormalities. Similar results were observed using the tubulin-GAL4 driver (data not shown).

To test whether Lapsyn is required for neuronal and/or synaptic function, we assayed mutant rescue with pre- and postsynaptic GAL4 drivers using the pan-neuronal $e l a v^{C 155}$-GAL4 driver and the Mhc-GAL4 muscle driver to express UAS-Lapsyn in homozygous Lapsyn ${ }^{z g 1}$ animals (Fig. 5D). Overexpression of UAS-Lapsyn driven by elav ${ }^{C 155}$-GAL4 resulted in partial rescue of $\operatorname{Lapsyn}^{z g 1}$ animals, with $34 \%$ surviving to the pupal stage. Animals rescued to the pupal stage by pan-neuronal expression failed to eclose as adults, indicating that Lapsyn likely has additional non-neuronal roles during pupation. Differences in the strength of transgene expression between actin-GAL4 and $e_{a v}{ }^{C 155}$-GAL4 could also contribute to the observed rescue effects. In contrast to ubiquitous or pan-neuronal expression of UAS-Lapsyn, animals rescued with the Mhc-GAL4 muscle driver died as embryos. This transgenic rescue analysis indicates that Lapsyn plays essential roles in the nervous system during embryonic and larval development, with additional non-neuronal roles likely during pupation. The failure to achieve complete rescue suggests that specific temporal and spatial regulation of Lapsyn may be required during development that is not recapitulated by GAL4-mediated expression.

Given that complete loss of Lapsyn resulted in embryonic lethality, we examined whether Lapsyn might play a role in regulating synaptic connectivity by analyzing 3rd instar larval NMJs in Lapsyn ${ }^{z g 1}$ heterozygotes. As Lapsyn is transcriptionally upregulated by increased neuronal activity, we hypothesized that a $50 \%$ reduction in gene expression levels might be detrimental to normal neuronal development. As a control, we used precise excision strains obtained from the P-element excision screen, as well as rescued animals in which UAS-Lapsyn was driven by

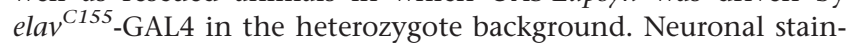
ing with anti-HRP revealed abnormal synaptic structure in $\operatorname{Lapsyn}^{z \mathrm{~g} 1} /+$ heterozygotes, with an increase in the formation of supernumerary satellite boutons (Fig. 6A,B). Satellite boutons form from increased branching of synaptic buds off of larger varicosities and are characteristically found with mutations in periactive zone proteins that regulate endocytosis or cell adhesion, resulting in dysfunctional synaptic growth-factor signaling (Dickman et al. 2006). A comparison of satellite bouton number in the control precise excision strain $\left(\operatorname{Lapsyn}^{P E} /+\right)$ vs. Lapsyn $n^{z g 1} /+$ heterozygotes was performed in segments A2-A5 at muscle 4 of 3rd instar larvae (Supplemental Fig. 6). Lapsyn ${ }^{\text {zg1 }} /+$ heterozygotes displayed 4.7-, 3.7-, 4.5-, and 4.3-fold increases in average satellite bouton numbers in segments A2-A5, respectively (Student's $t$-test $P<0.001$ ). Presynaptic expression of UAS-Lapsyn driven by elav ${ }^{C 155}$-GAL4 was able to rescue the satellite bouton phenotype in a dose-dependent manner (Fig. 6A,B), confirming that the phenotype was due to reduced Lapsyn expression. Similarly, the increase in satellite bouton formation observed in Lapsyn ${ }^{z g 1}$ heterozygotes was partially suppressed in the background of the rut ${ }^{1}$ mutant (Fig. 6C), where Lapsyn mRNA expression is up-regulated.

To test whether Lapsyn mutants might disrupt behavioral plasticity, we assayed associative learning in an established odorgustatory paradigm for larval associative learning (Neuser et al. 2005). Sugar-reward training in larvae can produce olfactory memories that require cAMP signaling (Honjo and FurukuboTokunaga 2005) and embryonic-born intrinsic Kenyon cells of the larval mushroom body (Pauls et al. 2010). We compared learning in behavioral assays where groups of 25-30 larvae were required to differentially associate two conditioned odors (isoamyl acetate vs. 1-octanol) with fructose as the reward stimuli. We first tested whether Lapsyn ${ }^{z g 1} /+$ heterozygotes showed normal olfactory and gustatory behaviors. No differences were found in taste and smell preference tests between controls and Lapsyn $^{z g 1} /+$ heterozygotes, as both groups showed significant attraction to the odors used and a preference for fructose over agar alone (Supplemental Fig. 7A-C). In contrast, associate learning in reciprocally trained groups was absent in Lapsyn ${ }^{z g 1} /+$ heterozygotes (learning index $[\mathrm{LI}]=-0.02 \pm 0.06, n=8, P<$ 0.01 ) compared with white (LI $=0.45 \pm 0.03, n=8$ ) or precise excision $\left(\right.$ Lapsyn $^{P E} /+; \mathrm{LI}=0.46 \pm 0.06, n=9$ ) controls (Fig. 6D). However, expression of a UAS-Lapsyn transgene driven by elav ${ }^{C 155}$-GAL4 was unable to rescue the learning defect in Lapsyn $^{\text {zg1 }} /+$ heterozygote larvae ( $\mathrm{LI}=0.05 \pm 0.01, n=7$ ) (Fig. $6 \mathrm{D})$. The lack of rescue may reflect additional defects unrelated to reduced Lapsyn function in the mutants, or the requirement for spatial, temporal or activity-regulated transcription of Lapsyn that is not reproduced by the elav $^{\mathrm{C} 155}$-GAL4 driver.

As Lapsyn was up-regulated by neuronal activity, we also tested whether increased expression of UAS-Lapsyn could alter synaptic development at larval NMJs. We overexpressed

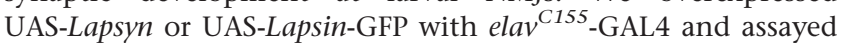
synaptic connectivity by anti-HRP and anti-Complexin immunostaining. In contrast to the increase in satellite bouton number in animals with reduced Lapsyn expression, presynaptic overexpression of Lapsyn resulted in a statistically significant increase in overall varicosity number, without the formation of satellite buttons (Fig. 7A,B). The results for overall varicosity number

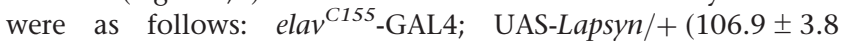
boutons, $n=12)$ vs. white $(80.4 \pm 4.3, \quad n=10, P<0.001)$; UAS-Lapsyn $/+(67 \pm 4.7, n=11, P<0.001)$; or elav ${ }^{C 155}$-GAL4 $/+$ $(94.2 \pm 4.3, n=12, P<0.05)$. Similar results were observed for overexpression of UAS-Lapsyn-GFP (data not shown). To test whether Lapsyn expression in the postsynaptic compartment could also stimulate synaptic growth, we overexpressed UAS-Lapsyn with the How ${ }^{24 B}$-GAL4 postsynaptic muscle driver. In contrast to the presynaptic effects of Lapsyn expression, postsynaptic expression of Lapsyn at the larval NMJ resulted in a statistically significant decrease in synaptic varicosity number (How ${ }^{24 B}$-GAL4; UAS-Lapysn ( $44.9 \pm 4.2$ boutons, $n=12$ ) vs. white $(80.4 \pm 4.3, n=10, P<0.001)$, UAS-Lapsyn $/+(67 \pm 4.7, n=11$, $P<0.001)$, or $H_{0 w}{ }^{24 B}$-GAL4 $/+(78.1 \pm 4.8, n=12 ; P<0.001)$ controls. These results suggest that Lapsyn expression is functionally coupled to changes in synaptic growth mechanisms at larval NMJs.

\section{Discussion}

Drosophila has proven to be a powerful model for identifying gene products involved in learning and memory based on olfactory, visual, and courtship behavioral assays (Waddell and Quinn 2001; Berry et al. 2008). How proteins identified in these studies regulate neuronal function or physiology to specifically alter behavioral plasticity is an ongoing area of investigation. Using 
A
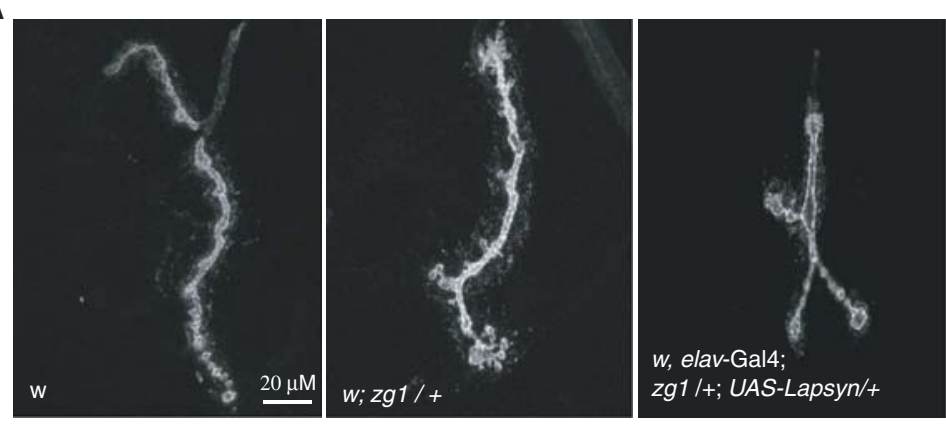

B
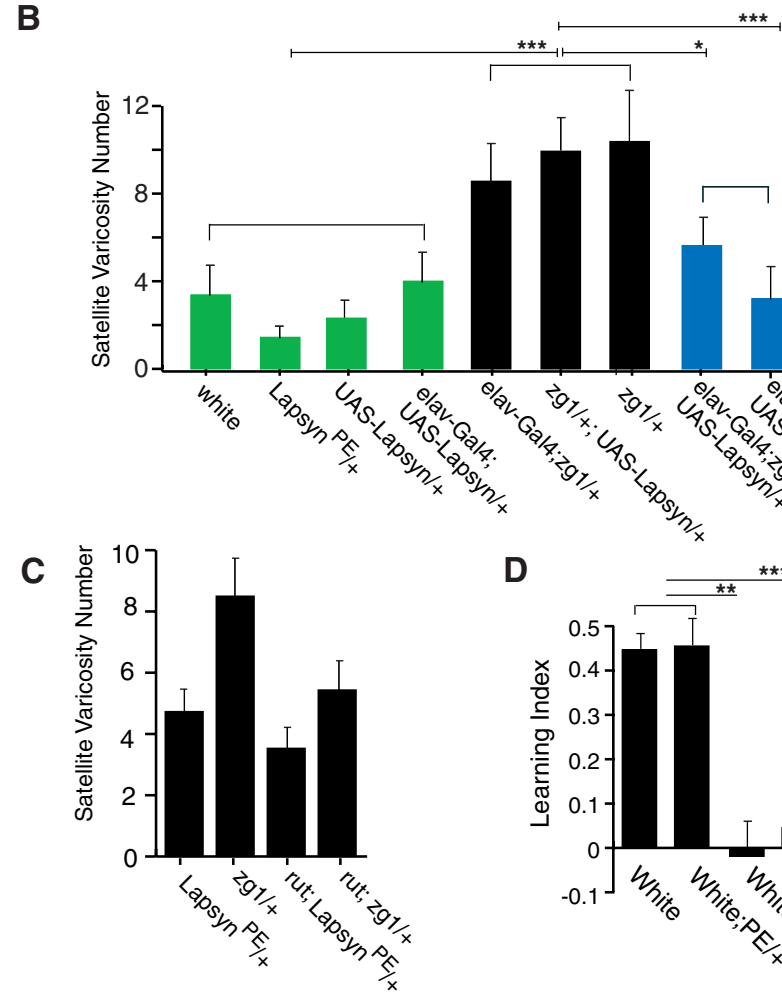

$* \star *$

D

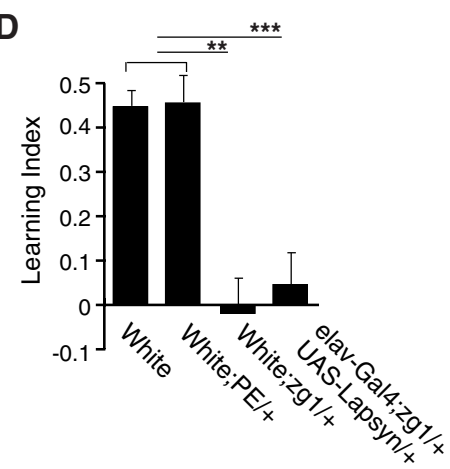

Figure 6. Lapsyn regulates synaptic growth and larval learning. (A) Representative NMJs for muscle 4 in segment $A 5$ of 3 rd instar larvae of the indicated genotypes immunostained with anti-HRP. (B) Quantification of satellite bouton number for the indicated genotypes at muscle fiber 4 in segment A5. Green bars represent controls, including white ( $3.4 \pm 1.3$ satellite boutons, $n=8)$, Lapsyn $n^{P E} /+$ $(1.4 \pm 0.6, n=8)$ and UAS-Lapsyn/+ $(2.3 \pm 0.8, n=8)$. Black bars represent heterozygous mutant Lapsyn $^{291} /+$ animals in different genetic backgrounds, including elav ${ }^{155}-G A L 4 ;$ Lapsyn $^{291} /+(8.5 \pm$ $1.8, n=7)$, Lapsyn $^{291} /+;$ UAS-Lapsyn/ $(9.9 \pm 1.5, n=7)$ and Lapsyn ${ }^{291} /+(10.4 \pm 2.3, n=8)$. Blue bars represent rescued animals expressing one or two alleles of transgenic UAS-Lapsyn driven by elav ${ }^{c 155}$-GAL4, including elav ${ }^{c 155}-$ GAL4; Lapsyn $^{291} /+$, UAS-Lapsyn $/+(5.6 \pm 1.2, \quad n=7)$, and elav ${ }^{155}$-GAL4; Lapsyn ${ }^{291} /+$; UAS-Lapsyn/UAS-Lapsyn (3.2 $\pm 1.6, n=7$ ). P-value of Student's $t$-test $\left.\left.{ }^{*}\right) P<0.05,\left(^{* * *}\right) P<0.001\right)$. Error bars represent SEM. (C) Satellite bouton overgrowth was partially reduced in heterozygous Lapsyn ${ }^{291} /+$ animals in the rut ${ }^{1}$ genetic background. Satellite bouton number was quantified on muscle 4 in segment $A 5$ of 3 rd instar larvae in rut ${ }^{1}$; Lapsyn $^{2 g 1} /+(5.4 \pm 0.13, n=16)$; rut $^{1} ; P E /+(3.5 \pm 0.78, n=15) ;$ Lapsyn $^{291} /+(8.5 \pm 0.02, n=16) ;$ and $P E /+(4.7 \pm 0.84, n=15)$. (D) Heterozygous Lapsyn ${ }^{291} /+$ larvae $(\mathrm{LI}=-0.02 \pm 0.06, n=8)$ fail to associate odor with fructose reward compared with white $(\mathrm{LI}=0.45 \pm 0.03, n=8, P<0.001)$ and white; $P E /+(\mathrm{LI}=0.46 \pm 0.06, n=9$, $P<0.01)$ controls. This defect was not significantly rescued by overexpression of UAS-Lapsyn driven using elav c155-GAL4 ( $\mathrm{LI}=0.05 \pm 0.01, n=7)$. Error bars represent SEM. $P$-values for Student's $t$-test $\left.\left({ }^{*}\right) P<0.05,(* *) P<0.01,(* * *) P<0.001\right)$.

the well-characterized 3rd instar larval NMJ as a model glutamatergic synapse, we compared the effects on synaptic connectivity of several learning mutants that alter cAMP signaling $\left(d n c^{1}, r u t^{1}\right.$, $a m n^{1}$ ) with the poorly characterized ARM mutant $r s h^{1}$. Each mutant altered synaptic connectivity at NMJs in a specific manner, suggesting that changes in neuronal connectivity in the CNS might contribute to the behavioral defects found in these strains. Our observations in $d n c^{1}$ and $r u t^{1}$ are similar to previous studies of synaptic morphology in these mutants (Zhong and Wu 1991; Renger et al. 2000). We also assayed gene expression in the mutants using microarray analysis, which revealed many neuronal transcripts that were transcriptionally altered. A longterm goal is to link transcriptional changes in specific loci to the behavioral and morphological defects found in learning and memory mutants.

Experimental approaches to define the biochemical transition from shortterm plasticity to long-term memory storage have suggested a key role for cAMP signaling. At the molecular level, one of the best-characterized pathways for STM has been described for gill withdrawal reflex facilitation in Aplysia. In this system, conditioned stimuli act through a serotonergic G proteincoupled receptor pathway to activate adenylyl cyclase in the presynaptic sensory neuron, resulting in the synthesis of cAMP (Cedar and Schwartz 1972; Brunelli et al. 1976). cAMP activates PKA, which phosphorylates a presynaptic potassium channel (Klein et al. 1982), leading to prolonged calcium influx and enhanced neurotransmitter release from the sensory neuron (Klein and Kandel 1980). Insights into the LLTM pathway in Aplysia have implicated CREB function. Robust training or stimulation with serotonin induces translocation of the catalytic subunit of PKA into the nucleus, where it activates the transcription factor CREB-1 and inhibits the transcriptional suppressor CREB-2 (Bartsch et al. 1995). CREB-1 acts on additional transcription factors to produce specific mRNAs that are transported to dendrites and captured by activated synapses. Local synthesis of new proteins and subsequent growth of synaptic connections is predicted to underlie long-term memory in the system (Dash et al. 1990; Bailey et al. 1992; Kaang et al. 1993; Casadio et al. 1999). It is likely that similar molecular pathways exist in other species. Transgenic Drosophila with inducible inhibition of PKA show memory impairment (Drain et al. 1991). PKA is also activated during hippocampal LTP induction in mammals (Roberson and Sweatt 1996), and transgenic mice that express an inhibitor of PKA have defective LTP and hippocampal-dependent memory (Huang et al. 1995; Abel et al. 1997), suggesting a general role for cAMP/PKA in the transition from learning to memory storage. 
A
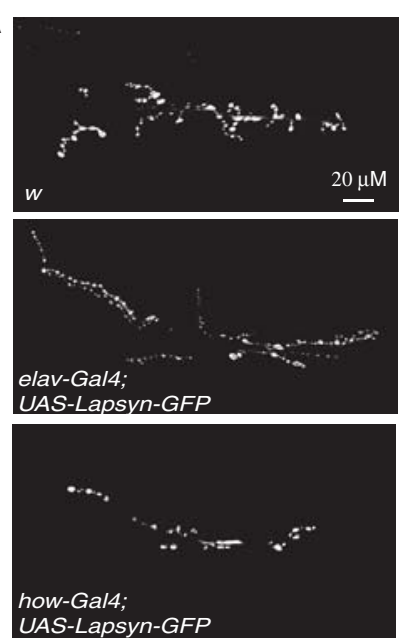

B

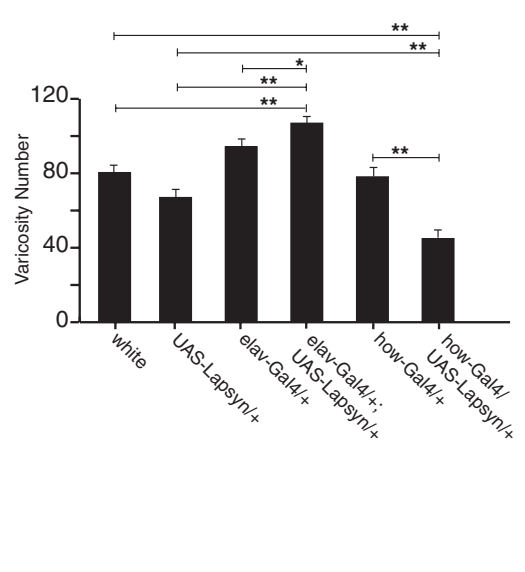

Figure 7. Lapsyn overexpression regulates synaptic growth. (A) Immunocytochemistry with anti-Complexin antiserum at muscles 6/7 NMJs in segment $A 3$ of Lapsyn overexpression lines of the indicated genotypes. $(B)$ Quantification of varicosity number for the indicated genotypes at muscles $6 / 7 \mathrm{NMJ}$ in segment A3. Overexpression of UAS-Lapsyn with the elav ${ }^{c 155}$-GAL4 driver increased varicosity number $(106.9 \pm 3.8, n=12)$ compared with white $(80.4 \pm 4.3, n=10)$, UAS-Lapsyn-GFP/+ $(67 \pm 4.7, n=11)$, and elav ${ }^{155}-G A L 4 /+(94.3 \pm 4.3, \quad n=12)$ controls. Overexpression of UAS-Lapsyn with the $\mathrm{How}^{24 B}$-GAL4 postsynaptic muscle driver resulted in a decrease in varicosity number $\left(44.9 \pm 4.2, n=12\right.$ ) compared with white, UAS-Lapsyn-GFP $/+$, and How ${ }^{24 B}$-GAL4/+ $(78.1 \pm 4.8, n=12)$ controls. Varicosity number was quantified for five to six larvae of each genotype. $P$-value of Student's $t$-test: $\left.\left(^{*}\right) P<0.05 ;\left(^{* *}\right) P<0.01\right)$. Error bars represent SEM.

In addition to CREB-dependent LLTM, which requires transcription and translation for its formation, the Radish-dependent ARM pathway represents a distinct long-term memory storage mechanism. These various memory pathways partially overlap in time. Three hours after training $\sim 50 \%$ of memory is stored as STM, with the rest present as ARM, which is formed immediately after training in flies and can last for days depending on training intensity (Quinn and Dudai 1976). ARM is not blocked by agents that disrupt electrical activity in the brain, suggesting that a biochemical pathway for ARM is likely initiated by learning stimuli, but does not require continued neuronal excitation for its expression. ARM is also not as sensitive to translation inhibition, as a $50 \%$ reduction of protein synthesis by cycloheximide does not affect ARM, but blocks LLTM (Tully et al. 1994).

Similar to the role of CREB in LLTM, Radish appears to be a key regulator of the ARM phase of memory. In contrast to the molecular pathways underlying STM (cAMP/PKA cascade) and LLTM (PKA/CREB), the signaling mechanisms mediating ARM are unknown. Unfortunately, the amino acid sequence of the radish locus gives little insight into its function, as it lacks known structural motifs or domains. Radish contains a serine/ arginine-rich sequence with very limited homology to splicing factors, hinting that it may be involved in RNA processing. The Radish protein also contains PKA phosphorylation sites and multiple NLS sites within its sequence. Consistent with these sequence features, we found that Radish is phosphorylated by PKA in vitro, linking ARM to the cAMP/PKA pathway. By generating a GFP-tagged Radish transgenic animal, we were able to characterize Radish localization. Radish was prominently localized to cell bodies of neurons in the CNS, but was enriched in the nucleus in other cell types such as salivary gland and muscle cells. Given the overlap between several of the NLS and PKA sites in Radish, it will be interesting to explore whether the phosphorylation state of Radish regulates its subcellular distribution. An attractive hypothesis is that activated PKA phosphorylates Radish at synapses, resulting in transport to the nucleus with accompanying effects on transcription or RNA processing that would modify long-term synaptic function. Given that ARM can last for days, a change in nuclear function is an attractive biological underpinning, even though ARM has been suggested to be a translation-independent form of memory (Tully et al. 1994). Given that general protein synthesis was reduced by only $50 \%$ in the previous studies, it is quite possible that ARM and LLTM have different thresholds for translational inhibition.

In terms of synaptic modifications in $r h^{1}$ mutants, we found that larval NMJ synapses were altered compared with controls. Specifically, $r s h^{1}$ mutants had shorter axonal projections onto target muscles and displayed more synaptic boutons within the innervated region. These alterations gave rise to a more compact innervation pattern than observed in controls. Overgrowth of synapses at larval NMJs was also observed in $d n c^{1}$ mutants, whereas reduced innervation length was found in $r u t^{1}$ mutants. As such, $r s h^{1}$ mutant NMJs display a unique phenotype compared with mutants that increase or decrease cAMP levels. The molecular mechanisms by which Radish regulates synaptic growth are unclear. Radish could directly interface with growth regulators at the synapse in a PKA-dependent fashion. Indeed, an interaction between Radish and Rac1 was found in a high-throughput yeast two-hybrid screen for interacting Drosophila proteins (Formstecher et al. 2005). Rac1 is a Rho family GTPase that regulates neuronal and synaptic morphology via reorganization of the cytoskeleton (Gao et al. 1999; Colley 2000). Rac1 function has also been linked to PAK1 and the Fragile-X Mental Retardation protein (FMRP), which alter synaptic and behavioral plasticity in mammals (Schenck et al. 2003; Hayashi et al. 2007). Recently, Rac activity has been linked to memory decay in Drosophila (Shuai et al. 2010), indicating that a Radish-Rac link might control memory processing via alterations in cytoskeletal modulation of synaptic function or stability. Although it is possible that Radish regulates synaptic properties through a Rac1 interaction, we were unable to observe a robust Rac1-Radish interaction in either yeast-two hybrid or GST pulldown experiments (data not shown). We also did not observe Radish-GFP enrichment at larval synapses where we quantified the synaptic growth defect, although the protein was present in larval axons. As such, it may be that NMJ defects in $r s h^{1}$ arise through downstream effects secondary to the loss of Radish function in a neuronal compartment besides the synapse.

To further explore this possibility and examine links between $r s h$ and the STM pathway, we performed genome-wide microarray studies on several learning and memory mutants. Although there were some shared transcriptional changes between $r s h^{1}$ and the other mutants $\left(d n c^{1}, r u t^{1}, a m n^{1}\right)$, most of the changes in $r h^{1}$ were unique. Although linking these changes to a direct effect on the underlying biology will require more work, several interesting loci were identified that could contribute to synaptic plasticity defects. The Drosophila NFAT homolog, a transcription factor that binds to the activity-regulated AP-1 (Fos/Jun) dimer, was robustly up-regulated by sevenfold in $r s h^{1}$ mutants. The RNA-binding protein smooth $(\mathrm{sm})$ was also up-regulated in $r s h^{1}$ mutants. Mutations in $s m$ have been shown to alter axonal pathfinding 
(Layalle et al. 2005). Other genes that were transcriptionally altered in $r s h^{1}$ mutants and that would be predicted to influence synaptic connectivity were the Sh potassium channel, the adapter protein Disabled, and the Lapsyn cell adhesion protein. We were intrigued by the potential role of Lapsyn, as LRR-containing proteins have been implicated in the regulation of neurite outgrowth and synapse formation. In particular, netrin-G ligand and synaptic-like adhesion molecule (SALM) are known LRR proteins that regulate neuronal connectivity and synapse formation (Lin et al. 2003; Kim et al. 2006; Wang et al. 2006). In Drosophila, LRR repeat proteins have been implicated in motor neuron target selection (Shinza-Kameda et al. 2006; Kurusu et al. 2008). Given the roles of other LRR-containing proteins in the regulation of neuronal connectivity, we explored whether Lapsyn might also function in this pathway. Lapsyn was up-regulated by neuronal activity in addition to being upregulated in $r s h^{1}$, making it an interesting transcriptional target to assay for a role in synaptic modification.

Lapsyn mRNA expression was broadly up-regulated in the brain by neuronal activity, suggesting a potential widespread effect on neuronal function. Lapsyn-GFP transgenic protein targeted to the presynaptic terminal, partially overlapping with the periactive zone, a region of the nerve terminal enriched in proteins that regulate synaptic vesicle endocytosis and synaptic connectivity. Animals lacking Lapsyn died at the end of embryogenesis, although the early stages of nervous system formation appeared normal. We were able to partially rescue Lapsyn mutants with neuronal expression of a Lapsyn transgene, indicating an essential function for the protein in the nervous system. Rescue to adulthood required expression outside the nervous system, suggesting Lapysn is likely to have functions in other tissue types as well. Manipulations of Lapsyn expression in the nervous system resulted in distinct defects in synaptic connectivity at the NMJ. Heterozygotes expressing only a single copy of the Lapsyn gene displayed supernumerary satellite bouton formation, a phenotype commonly associated with mutants that disrupt synaptic endocytosis (Dickman et al. 2006) or that alter the transmission or trafficking of synaptic growth factors through the endosomal system (O'Connor-Giles et al. 2008; Rodal et al. 2008). This increase in satellite boutons in Lapsyn heterozygotes suggests that the protein plays a role in the regulation of synaptic growth signaling. Overexpression of Lapsyn, as induced by activity or observed in $r s h^{1}$ mutants, also elicited a change in synaptic growth, resulting in an increase in overall bouton number at larval NMJs. Thus, regulation of Lapsyn levels modulate synaptic growth mechanisms at NMJs. Lapsyn mutant heterozygotes also display defects in larval associative learning, although we were unable to rescue this phenotype with pan-neuronal overexpression. The lack of a specific rescue makes it unclear whether the learning defects are linked to a non-Lapsyn function, or if a more specific spatial and temporal expression of Lapsyn is required for functional rescue.

How Lapsyn participates in synaptic signaling is currently unclear. The closest mammalian homologs of Lapsyn are the NGL family of synaptic adhesion molecules (Woo et al. 2009). Three isoforms are found in mammals, NGL-1, NGL-2, and NGL-3, which interact with netrin-G1, netrin-G2, and the receptor tyrosine phosphatase LAR, respectively. NGL-1 promotes axonal outgrowth (Lin et al. 2003), whereas NGL-2 is capable of triggering synapse formation (Biederer 2006; Kim et al. 2006). The interaction of NGL-3 with LAR is intriguing, as the Drosophila LAR homolog has been shown to bind the heparan sulfate proteoglycans Syndecan and Dallylike to regulate synaptic growth at the NMJ (Johnson et al. 2006). The homology between Lapsyn and the mammalian NLG family is restricted to the extracellular LRR domain, with no homology observed in the intracellular C terminus. The three mammalian NLGs also lack homology to each other at the $\mathrm{C}$ terminus, except for the presence of a PDZ-binding domain at the end of the intracellular domain. It will be important to identify binding partners for Lapsyn at the synapse to define how it may regulate synaptic adhesion or signaling between the pre- and postsynaptic compartments to regulate synaptic growth. Likewise, additional studies into the Radishdependent ARM phase of memory may reveal how $r$ sh-dependent changes in Lapsyn levels contribute to the synaptic and behavioral defects of this memory mutant.

\section{Materials and Methods}

\section{Drosophila genetics}

Drosophila were cultured on standard medium at $22^{\circ} \mathrm{C}$. The rut ${ }^{1}$, $d n c^{1}, a m n^{1}$, and $r s h^{1}$ mutants were outcrossed into the CS genetic background, with CS serving as a control for experimental manipulations.

\section{Western and immunocytochemical analysis}

Anti-Lapsyn antiserum was generated in rabbits immunized with a recombinant protein encompassing the extracellular domain of Lapsyn (from serine 58 to phenylalanine 275) fused to GST (Invitrogen). Anti-Lapsyn was used at a 1:300 dilution and detected using goat anti-rabbit antiserum conjugated to Alexa Fluor 680 (Invitrogen, \#A21076). Anti-GFP antibodies (Invitrogen, \#A11120) were used at 1:5000 and detected using goat anti-mouse antiserum conjugated to IRDye800 (Rockland, \#610-132-121). Anti-Complexin (Huntwork and Littleton 2007) antiserum was used at 1:1000 and detected using goat anti-rabbit antiserum conjugated to Alexa Fluor 680 (Invitrogen, \#A21076). Cell nuclei were detected with SYTO Orange (Molecular Probes) at 1:1000. Western blot analysis was done using a LICOR Odyssey infrared scanner (LICOR).

\section{Morphological analysis}

Immunostaining was performed on wandering 3rd instar larvae or 0-22-h staged embryos at room temperature as indicated. 3rd instar larvae were dissected in Drosophila HL3.1 physiological saline and fixed in $4 \%$ formaldehyde for $45 \mathrm{~min}$ before staining with anti-Syt1 (Littleton et al. 1993) antiserum at 1:1000, anti-Complexin antiserum at 1:500, or goat anti-HRP antiserum conjugated to DyLight 549 (Jackson ImmunoResearch). Immunoreactive proteins were visualized on a Zeiss Pascal confocal microscope. Rhodamine-phalloidin (Invitrogen) was used at 1:250 to stain muscles for surface area measurements. DAPI immunostaining was conducted using Vectashield mounting medium (Vector Laboratories) Confocal images were quantified for varicosity number, synapse branch number (branches with at least five boutons per branch), innervation length along the muscle (measured as the distance between the most distal boutons on muscles 6 and 7), muscle length, and muscle width. All error measurements are SEM. Anti-Fas2 antiserum (Developmental Studies Hybridoma Bank) was used at 1:1000 and anti-bruchpilot (nc82, Developmental Studies Hybridoma Bank) antiserum was used at 1:50. For embryonic immunostaining, embryos were dechorionated with $50 \%$ bleach and fixed in $4 \%$ formaldehyde for $20 \mathrm{~min}$. Embyros were then devitellinized and stained with anti-Fas2 antiserum at 1:200.

\section{Kinase assay}

The Radish kinase assay was performed in triplicate using the catalytic subunit of PKA (New England Biolabs) according to the manufacturer's instructions, with the addition of $\left[\gamma^{-}{ }^{32} \mathrm{P}\right] \mathrm{ATP}$ (Perkin Elmer). Approximately $10 \mu \mathrm{g}$ of purified GST-fusion protein was used per reaction and incubated with 2500 units of recombinant PKA. Reaction products were separated by SDS-PAGE, and the gels were stained with Bio-Safe Coomassie 
(Bio-Rad), dried, and exposed to autoradiography film for $30 \mathrm{~min}$ at room temperature.

\section{Microarray analysis}

Microarray analysis was performed using Affymetrix Drosophila Genechips with biotinylated cRNA by the methods described in the Affymetrix Genechip expression manual as previously described (Guan et al. 2005). RNA was isolated from the heads of adult male flies aged 3-4 d post-eclosion, at room temperature. All flies were sacrificed between 2 and 4 PM to reduce any circadian transcriptional changes. For a single chip, 600 heads were sufficient to obtain $150 \mathrm{mg}$ of total RNA, $3 \mathrm{mg}$ of mRNA, and $45 \mathrm{mg}$ of cRNA/1 mg mRNA. Total RNA was isolated using Trizol (GIBCO BRL). Biotin-labeled cRNA was generated with an RNA Transcript Labeling Kit (Enzo) using the supplied protocol with the modification of running half reaction mixes. The Affymetrix array high-density oligonucleotide arrays were probed, hybridized, stained, and washed in MIT's Biopolymers Facility according to the manufacturer's instructions. Microarray analysis was performed using Affymetrix's Microarray Suite MAS 5.0 statisticsbased analysis software, Student's t-test, and Mann-Whitney $U$-test using the following normalization values: Scaling target: 1500, Alpha1: 0.04, Alpha2: 0.06, Tau: 0.015, Gamma1L: 0.0025, Gamma1H: 0.0025, Gamma2L: 0.0030, Gamma2H: 0.0030 and Perturbation: 1.1. Gene changes were called based upon the stringency criterion that differential regulation must be reported by the Affymetrix software for at least $75 \%$ of the pairwise comparisons. A summary of the expression levels of each gene is provided based on Affymetrix detection algorithms (Supplemental Table 1), allowing additional analysis with other microarray tools.

\section{Quantitative real-time RT-PCR and semi-quantitative RT-PCR}

Quantitative real-time RT-PCR and semi-quantitative RT-PCR were performed as previously described (Guan et al. 2005). Primers were made to sequences used in the design of probe pairs by Affymetrix and each gene ORF. cDNA was diluted into equivalent amounts from $1 \mathrm{mg}$ of total RNA per $1 \mathrm{~mL}$. cDNAs were analyzed by quantitative real-time PCR using a SYBR Green PCR amplification kit (Applied Biosystems) and measured in a Bio-Rad iCycler (Bio-Rad, Richmond, CA). Target gene message levels were normalized to glyceraldehyde-3-phosphate dehydrogenase levels and then to the control sample. All PCRs were performed in triplicate. The primers used for semi-quantitative RT-PCR were as follows:

GAPDH1 (forward: 5'-aatcaaggctaaggtcgaggag-3' /reverse: $5^{\prime}$-taaccgaactcgttgtcgtacc-3')

Syt7 (forward: 5'-ggaaggaacgagttaatagg-3'/reverse: $5^{\prime}$-tactcaggttttaagcgatgcc- $\left.3^{\prime}\right)$

Nmda1 (forward: $5^{\prime}$-aatattgtttgcagcttaatcg-3' $/$ reverse: $5^{\prime}$-gttcatatagccttagttgac-3')

Act88F (forward: 5'-ggacagtgatagacaaaagc-3' / reverse: $5^{\prime}$-gtaaaaatccgctataccgc-3')

CG7607 (forward: 5'-cacgaatctcacatagaagcg-3' / reverse: $5^{\prime}$-tcagctttactgtgtcaaagg-3')

$R g l$ (forward: $5^{\prime}$-actctcagtctatgggaaatg-3' / reverse: $5^{\prime}$-gacctgcgccaaagtaaacc-3')

aay (forward: $5^{\prime}$-atagcgacgattccctaatc- $3^{\prime}$ /reverse: $5^{\prime}$-actaacagtgcgcgcttaac-3')

mthl8 (forward: $5^{\prime}$-caccttaactacgctgtaag- $3^{\prime}$ /reverse: $5^{\prime}$-cacagatattgtcgatacac- $\left.3^{\prime}\right)$

5HT-7 (forward: $5^{\prime}$-cctctaaatgtaacttgttgttg- $3^{\prime}$ /reverse: $5^{\prime}$-cggtgctgattgttttcgaaac- $3^{\prime}$ )

Lapsyn (forward: $5^{\prime}$-cttctgcgtgatacactgtag- $3^{\prime} /$ reverse: $5^{\prime}$-ctattttcgggcaatatcacag- $\left.3^{\prime}\right)$

glec (forward: 5'-acggtccaaaaatgttgttgc- $3^{\prime}$ /reverse: $5^{\prime}$-ctgtcgatcgcttgtctaac- $\left.3^{\prime}\right)$.

\section{In situ hybridization to brain sections}

Dig-11-UTP-labeled antisense and sense RNA probes were prepared using T7 polymerase (Ambion). Adult Drosophila were placed into fly collars after $\mathrm{CO}_{2}$ anesthesia and embedded in frozen OCT on dry ice, and $16-\mu \mathrm{m}$ tissue sections were collected on a cryostat (Leica) and dried for $30 \mathrm{~min}$ to $3 \mathrm{~h}$ at room temperature. The sections were processed as described previously (Nighorn et al. 1991), except for the in situ hybridization temperature $\left(63^{\circ} \mathrm{C}\right)$. The primers used to make in situ hybridization probes by PCR, which also included the T7 RNA polymerase promoter at each end (data not shown) were as follows:

mthl8 (forward: 5'-gcagttctgcattcttggag-3' / reverse: $5^{\prime}$-gccaaaacatcagttttgtg-3')

Lapsyn (forward: $5^{\prime}$-ctgcattggcagctgctgctgacgttc-3' / reverse: $5^{\prime}$-gcagccagctccaaagagccag-3').

\section{Appetitive olfactory learning assays in Drosophila larvae}

3rd instar feeding stage larvae aged 4-5 d after egg laying were used in learning assays. Animals were kept in a $25^{\circ} \mathrm{C}$ incubator with a $12 \mathrm{~h}: 12 \mathrm{~h}$ light-dark cycle, and experiments were performed under regular light in a fume hood at $25^{\circ} \mathrm{C}$. Learning assays were performed en masse using published protocols (Neuser et al. 2005) with a few modifications. Groups of 25-40 larvae were assayed after either of two reciprocal training regimes: (1) larvae were exposed to isoamyl acetate (IAA) (MP Biomedical, cat no. 155077, diluted 1:50 in paraffin oil) with a positive reinforcer of 2M D-fructose (FRU) (Alfa Aesar, A17718); or 1-octanol (OCT) (MP Biomedical, cat no. 155969) without reinforcer (IAA+ /OCT). (2) Alternatively, larvae were trained reciprocally (IAA/ OCT + ). Associative learning index (LI) was calculated as: $\mathrm{LI}=$ $\left(\mathrm{PREF}_{\text {ind }} \mathrm{IAA}_{+} / \mathrm{OCT}^{-}-\mathrm{PREF}_{\text {ind }} \mathrm{IAA} / \mathrm{OCT}+\right) / 2 ; \mathrm{PREF}_{\text {ind }}=($ number of observations IAA-number of observations ${ }_{\mathrm{OCT}}$ )/number of

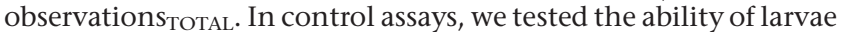
to detect IAA, OCT, and FRU. Briefly, naïve animals were given the choice between either paraffin-diluted IAA vs. paraffin (PREF IAA), or between undiluted OCT vs. an empty container (PREF OCT). To assay FRU detection, naïve animals were given the choice between one side of an agarose plate containing pure $1 \%$ agarose on one half vs. $2 \mathrm{M}$ FRU in 1\% agarose (PREF FRU) on the other half. For all tests, animals were assayed in groups of 20-40 larvae, with the calculation of PREF values as: number of observations $s_{\mathrm{ODOR}}$ or FRU/number of observations TOTAL. $_{\text {. }}$.

\section{Acknowledgments}

We thank Elisabeth Folkers for helpful discussions, Lei Xu and Xiaohu Zhao for technical support, MIT's Biopolymer and BioMicroCenter for assistance with microarray chip hybridizations and data analysis, and the Bloomington and Vienna Stock Centers for Drosophila stocks. This work was supported by grant no. NS43244 from the NIH to J.T.L.

\section{References}

Abel T, Nguyen PV, Barad M, Deuel TA, Kandel ER, Bourtchouladze R. 1997. Genetic demonstration of a role for PKA in the late phase of LTP and in hippocampus-based long-term memory. Cell 88: 615-626.

Baeg GH, Zhou R, Perrimon N. 2005. Genome-wide RNAi analysis of JAK/STAT signaling components in Drosophila. Genes Dev 19: $1861-1870$

Bailey CH, Montarolo P, Chen M, Kandel ER, Schacher S. 1992. Inhibitors of protein and RNA synthesis block structural changes that accompany long-term heterosynaptic plasticity in Aplysia. Neuron 9: 749-758.

Bartsch D, Ghirardi M, Skehel PA, Karl KA, Herder SP, Chen M, Bailey CH, Kandel ER. 1995. Aplysia CREB2 represses long-term facilitation: Relief of repression converts transient facilitation into long-term functional and structural change. Cell 83: 979-992.

Bejarano F, Luque CM, Herranz H, Sorrosal G, Rafel N, Pham TT, Milan M. 2008. A gain-of-function suppressor screen for genes involved in dorsal-ventral boundary formation in the Drosophila wing. Genetics 178: $307-323$. 
Berry J, Krause WC, Davis RL. 2008. Olfactory memory traces in Drosophila. Prog Brain Res 169: 293-304.

Biederer T. 2006. Hooking up new synapses. Nat Neurosci 9: 1203-1204.

Brunelli M, Castellucci V, Kandel ER. 1976. Synaptic facilitation and behavioral sensitization in Aplysia: Possible role of serotonin and cyclic AMP. Science 194: 1178-1181.

Budnik V, Zhong Y, Wu CF. 1990. Morphological plasticity of motor axons in Drosophila mutants with altered excitability. J Neurosci 10: 3754-3768.

Byers D, Davis RL, Kiger JA Jr. 1981. Defect in cyclic AMP phosphodiesterase due to the dunce mutation of learning in Drosophila melanogaster. Nature 289: 79-81.

Cantera R, Kozlova T, Barillas-Mury C, Kafatos FC. 1999. Muscle structure and innervation are affected by loss of Dorsal in the fruit fly, Drosophila melanogaster. Mol Cell Neurosci 13: 131-141.

Casadio A, Martin KC, Giustetto M, Zhu H, Chen M, Bartsch D, Bailey CH, Kandel ER. 1999. A transient, neuron-wide form of CREB-mediated long-term facilitation can be stabilized at specific synapses by local protein synthesis. Cell 99: 221-237.

Castillo PE, Schoch S, Schmitz F, Sudhof TC, Malenka RC. 2002. RIM1 $\alpha$ is required for presynaptic long-term potentiation. Nature 415: 327-330.

Cedar H, Schwartz JH. 1972. Cyclic adenosine monophosphate in the nervous system of Aplysia californica. II. Effect of serotonin and dopamine. J Gen Physiol 60: 570-587.

Chen CN, Denome S, Davis RL. 1986. Molecular analysis of cDNA clones and the corresponding genomic coding sequences of the Drosophila dunce+ gene, the structural gene for cAMP phosphodiesterase. Proc Natl Acad Sci 83: 9313-9317.

Clemens JC, Worby CA, Simonson-Leff N, Muda M, Maehama T, Hemmings BA, Dixon JE. 2000. Use of double-stranded RNA interference in Drosophila cell lines to dissect signal transduction pathways. Proc Natl Acad Sci 97: 6499-6503.

Colley NJ. 2000. Cell biology. Actin' up with Rac1. Science 290: 1902-1903.

Cowan TM, Siegel RW. 1986. Drosophila mutations that alter ionic conduction disrupt acquisition and retention of a conditioned odor avoidance response. J Neurogenet 3: 187-201.

Dash PK, Hochner B, Kandel ER. 1990. Injection of the cAMP-responsive element into the nucleus of Aplysia sensory neurons blocks long-term facilitation. Nature 345: 718-721.

Dason JS, Romero-Pozuelo J, Marin L, Iyengar BG, Klose MK, Ferrus A, Atwood HL. 2009. Frequenin/NCS-1 and the Ca2+-channel $\alpha 1$-subunit co-regulate synaptic transmission and nerve-terminal growth. J Cell Sci 122(Pt 22): 4109-4121.

Davis GW, Schuster CM, Goodman CS. 1996. Genetic dissection of structural and functional components of synaptic plasticity. III. CREB is necessary for presynaptic functional plasticity. Neuron 17: $669-679$.

Delalle I, Pfleger CM, Buff E, Lueras P, Hariharan IK. 2005. Mutations in the Drosophila orthologs of the F-actin capping protein $\alpha$ - and $\beta$-subunits cause actin accumulation and subsequent retinal degeneration. Genetics 171: $1757-1765$.

DeZazzo J, Sandstrom D, de Belle S, Velinzon K, Smith P, Grady L, DelVecchio M, Ramaswami M, Tully T. 2000. nalyot, a mutation of the Drosophila myb-related Adf1 transcription factor, disrupts synapse formation and olfactory memory. Neuron 27: 145-158.

Dickman DK, Lu Z, Meinertzhagen IA, Schwarz TL. 2006. Altered synaptic development and active zone spacing in endocytosis mutants. Curr Biol 16: $591-598$.

Didelot G, Molinari Fl, Tchenio P, Comas D, Milhiet E, Munnich A, Colleaux L, Preat T. 2006. Tequila, a neurotrypsin ortholog, regulates long-term memory formation in Drosophila. Science 313: 851-853.

Drain P, Folkers E, Quinn WG. 1991. cAMP-dependent protein kinase and the disruption of learning in transgenic flies. Neuron 6: 71-82.

Dubnau J, Tully T. 1998. Gene discovery in Drosophila: New insights for learning and memory. Annu Rev Neurosci 21: 407-444.

Dudai Y, Jan YN, Byers D, Quinn WG, Benzer S. 1976. dunce, a mutant of Drosophila deficient in learning. Proc Natl Acad Sci 73: 1684-1688.

Fan H, Sakuraba K, Komuro A, Kato S, Harada F, Hirose Y. 2003. PCIF1, a novel human WW domain-containing protein, interacts with the phosphorylated RNA polymerase II. Biochem Biophys Res Commun 301: $378-385$.

Feany MB, Quinn WG. 1995. A neuropeptide gene defined by the Drosophila memory mutant amnesiac. Science 268: 869-873.

Folkers E, Drain P, Quinn WG. 1993. Radish, a Drosophila mutant deficient in consolidated memory. Proc Natl Acad Sci 90: 8123-8127.

Folkers E, Waddell S, Quinn WG. 2006. The Drosophila radish gene encodes a protein required for anesthesia-resistant memory. Proc Natl Acad Sci 103: $17496-17500$

Formstecher E, Aresta S, Collura V, Hamburger A, Meil A, Trehin A, Reverdy C, Betin V, Maire S, Brun C, et al. 2005. Protein interaction mapping: A Drosophila case study. Genome Res 15: 376-384.
Ganetzky B, Wu CF. 1982. Drosophila mutants with opposing effects on nerve excitability: Genetic and spatial interactions in repetitive firing. J Neurophysiol 47: 501-514.

Gao FB, Brenman JE, Jan LY, Jan YN. 1999. Genes regulating dendritic outgrowth, branching, and routing in Drosophila. Genes Dev 13: $2549-2561$.

Gaul U, Mardon G, Rubin GM. 1992. A putative Ras GTPase activating protein acts as a negative regulator of signaling by the Sevenless receptor tyrosine kinase. Cell 68: 1007-1019.

Gertler FB, Hill KK, Clark MJ, Hoffmann FM. 1993. Dosage-sensitive modifiers of Drosophila abl tyrosine kinase function: prospero, a regulator of axonal outgrowth, and disabled, a novel tyrosine kinase substrate. Genes Dev 7: 441-453.

Goldstein SA, Price LA, Rosenthal DN, Pausch MH. 1996. ORK1, a potassium-selective leak channel with two pore domains cloned from Drosophila melanogaster by expression in Saccharomyces cerevisiae. Proc Natl Acad Sci 93: 13256-13261.

Guan Z, Saraswati S, Adolfsen B, Littleton JT. 2005. Genome-wide transcriptional changes associated with enhanced activity in the Drosophila nervous system. Neuron 48: 91-107.

Gunawardane RN, Martin OC, Cao K, Zhang L, Dej K, Iwamatsu A, Zheng Y. 2000. Characterization and reconstitution of Drosophila $\gamma$-tubulin ring complex subunits. J Cell Biol 151: 1513-1524.

Hayashi ML, Rao BS, Seo JS, Choi HS, Dolan BM, Choi SY, Chattarji S, Tonegawa S. 2007. Inhibition of p21-activated kinase rescues symptoms of fragile X syndrome in mice. Proc Natl Acad Sci 104: $11489-11494$.

Hodge JJ, Mullasseril P, Griffith LC. 2006. Activity-dependent gating of CaMKII autonomous activity by Drosophila CASK. Neuron 51: $327-337$.

Honjo K, Furukubo-Tokunaga K. 2005. Induction of cAMP response element-binding protein-dependent medium-term memory by appetitive gustatory reinforcement in Drosophila larvae. J Neurosci 25: $7905-7913$.

Howard L, Nelson KK, Maciewicz RA, Blobel CP. 1999. Interaction of the metalloprotease disintegrins MDC9 and MDC15 with two SH3 domain-containing proteins, endophilin I and SH3PX1. J Biol Chem 274: $31693-31699$.

Huang YY, Kandel ER, Varshavsky L, Brandon EP, Qi M, Idzerda RL, McKnight GS, Bourtchouladze R. 1995. A genetic test of the effects of mutations in PKA on mossy fiber LTP and its relation to spatial and contextual learning. Cell 83: 1211-1222.

Huang CH, Lin TY, Pan RL, Juang JL. 2007. The involvement of Abl and PTP61F in the regulation of Abi protein localization and stability and lamella formation in Drosophila S2 cells. J Biol Chem 282: 32442-32452.

Huntwork S, Littleton JT. 2007. A complexin fusion clamp regulates spontaneous neurotransmitter release and synaptic growth. Nat Neurosci 10: 1235-1237.

Jan YN, Jan LY, Dennis MJ. 1977. Two mutations of synaptic transmission in Drosophila. Proc R Soc Lond B Biol Sci 198: 87-108.

Johnson KG, Tenney AP, Ghose A, Duckworth AM, Higashi ME, Parfitt K, Marcu O, Heslip TR, Marsh JL, Schwarz TL, et al. 2006. The HSPGs Syndecan and Dallylike bind the receptor phosphatase LAR and exert distinct effects on synaptic development. Neuron 49: 517-531.

Kaang BK, Kandel ER, Grant SG. 1993. Activation of cAMP-responsive genes by stimuli that produce long-term facilitation in Aplysia sensory neurons. Neuron 10: 427-435.

Kim S, Burette A, Chung HS, Kwon SK, Woo J, Lee HW, Kim K, Kim H, Weinberg RJ, Kim E. 2006. NGL family PSD-95-interacting adhesion molecules regulate excitatory synapse formation. Nat Neurosci 9: $1294-1301$.

Klein M, Kandel ER. 1980. Mechanism of calcium current modulation underlying presynaptic facilitation and behavioral sensitization in Aplysia. Proc Natl Acad Sci 77: 6912-6916.

Klein M, Camardo J, Kandel ER. 1982. Serotonin modulates a specific potassium current in the sensory neurons that show presynaptic facilitation in Aplysia. Proc Natl Acad Sci 79: 5713-5717.

Krupinski J, Coussen F, Bakalyar HA, Tang WJ, Feinstein PG, Orth K, Slaughter C, Reed RR, Gilman AG. 1989. Adenylyl cyclase amino acid sequence: Possible channel- or transporter-like structure. Science 244: $1558-1564$.

Kurten RC, Cadena DL, Gill GN. 1996. Enhanced degradation of EGF receptors by a sorting nexin, SNX1. Science 272: 1008-1010.

Kurusu M, Cording A, Taniguchi M, Menon K, Suzuki E, Zinn K. 2008. A screen of cell-surface molecules identifies leucine-rich repeat proteins as key mediators of synaptic target selection. Neuron 59: $972-985$

Layalle S, Coessens E, Ghysen A, Dambly-Chaudiere C. 2005. Smooth, a hnRNP encoding gene, controls axonal navigation in Drosophila. Genes Cells 10: 119-125. 
Levin LR, Han PL, Hwang PM, Feinstein PG, Davis RL, Reed RR. 1992. The Drosophila learning and memory gene rutabaga encodes a $\mathrm{Ca} 2+/$ Calmodulin-responsive adenylyl cyclase. Cell 68: 479-489.

Liebl FL, Werner KM, Sheng Q, Karr JE, McCabe BD, Featherstone DE. 2006. Genome-wide P-element screen for Drosophila synaptogenesis mutants. J Neurobiol 66: 332-347.

Lin JC, Ho WH, Gurney A, Rosenthal A. 2003. The netrin-G1 ligand NGL-1 promotes the outgrowth of thalamocortical axons. Nat Neurosci 6: $1270-1276$.

Littleton JT, Stern M, Schulze K, Perin M, Bellen HJ. 1993. Mutational analysis of Drosophila synaptotagmin demonstrates its essential role in $\mathrm{Ca}(2+)$-activated neurotransmitter release. Cell 74: 1125-1134.

Livingstone MS, Sziber PP, Quinn WG. 1984. Loss of calcium/calmodulin responsiveness in adenylate cyclase of rutabaga, a Drosophila learning mutant. Cell 37: 205-215.

Lu CS, Hodge JJ, Mehren J, Sun XX, Griffith LC. 2003. Regulation of the $\mathrm{Ca} 2+/ \mathrm{CaM}$-responsive pool of CaMKII by scaffold-dependent autophosphorylation. Neuron 40: 1185-1197.

Moore MS, DeZazzo J, Luk AY, Tully T, Singh CM, Heberlein U. 1998. Ethanol intoxication in Drosophila: Genetic and pharmacological evidence for regulation by the cAMP signaling pathway. Cell 93: 997-1007.

Neuser K, Husse J, Stock P, Gerber B. 2005. Appetitive olfactory learning in Drosophila larvae: Effects of repetition, reward strength, age, gender, assay type, and memory span. Anim Behav 69: 891-898.

Nicolai M, Lasbleiz C, Dura JM. 2003. Gain-of-function screen identifies a role of the Src64 oncogene in Drosophila mushroom body development. J Neurobiol 57: 291-302.

Nighorn A, Healy MJ, Davis RL. 1991. The cyclic AMP phosphodiesterase encoded by the Drosophila dunce gene is concentrated in the mushroom body neuropil. Neuron 6: 455-467.

O'Connor-Giles KM, Ho LL, Ganetzky B. 2008. Nervous wreck interacts with thickveins and the endocytic machinery to attenuate retrograde BMP signaling during synaptic growth. Neuron 58: 507-518.

Parrish JZ, Kim MD, Jan LY, Jan YN. 2006. Genome-wide analyses identify transcription factors required for proper morphogenesis of Drosophila sensory neuron dendrites. Genes Dev 20: 820-835.

Pawson C, Eaton BA, Davis GW. 2008. Formin-dependent synaptic growth: Evidence that Dlar signals via Diaphanous to modulate synaptic actin and dynamic pioneer microtubules. J Neurosci 30: 10655-10666.

Pauls D, Selcho M, Gendre N, Stocker RF, Thum AS. 2010. Drosophila larvae establish appetitive olfactory memories via mushroom body neuorns of embryonic origin. J Neurosci 28: 11111-11123.

Quinn WG, Dudai Y. 1976. Memory phases in Drosophila. Nature 262: 576-577.

Quinn WG, Sziber PP, Booker R. 1979. The Drosophila memory mutant amnesiac. Nature 277: 212-214.

Renger JJ, Ueda A, Atwood HL, Govind CK, Wu CF. 2000. Role of cAMP cascade in synaptic stability and plasticity: Ultrastructural and physiological analyses of individual synaptic boutons in Drosophila memory mutants. J Neurosci 20: 3980-3992.

Roberson ED, Sweatt JD. 1996. Transient activation of cyclic AMP-dependent protein kinase during hippocampal long-term potentiation. J Biol Chem 271: 30436-30441.
Rodal AA, Motola-Barnes RN, Littleton JT. 2008. Nervous wreck and Cdc42 cooperate to regulate endocytic actin assembly during synaptic growth. J Neurosci 28: 8316-8325.

Schenck A, Bardoni B, Langmann C, Harden N, Mandel JL, Giangrande A. 2003. CYFIP/Sra-1 controls neuronal connectivity in Drosophila and links the Rac1 GTPase pathway to the fragile X protein. Neuron 38: 887-898.

Schwaerzel M, Jaeckel A, Mueller U. 2007. Signaling at A-kinase anchoring proteins organizes anesthesia-sensitive memory in Drosophila. J Neurosci 27: 1229-1233.

Shinza-Kameda M, Takasu E, Sakurai K, Hayashi S, Nose A. 2006. Regulation of layer-specific targeting by reciprocal expression of a cell adhesion molecule, capricious. Neuron 49: 205-213.

Shuai Y, Lu B, Hu Y, Wang L, Sun K, Zhong Y. 2010. Forgetting is regulated through Rac activity in Drosophila. Cell 140: 579-589.

Simpson CL, Lemmens R, Miskiewicz K, Broom WJ, Hansen VK, van Vught PW, Landers JE, Sapp P, Van Den Bosch L, Knight J, et al. 2009. Variants of the elongator protein 3 (ELP3) gene are associated with motor neuron degeneration. Hum Mol Genet 18: 472-481.

Skoulakis EM, Kalderon D, Davis RL. 1993. Preferential expression in mushroom bodies of the catalytic subunit of protein kinase A and its role in learning and memory. Neuron 11: 197-208.

Tully T, Preat T, Boynton SC, Del Vecchio M. 1994. Genetic dissection of consolidated memory in Drosophila. Cell 79: 35-47.

Waddell S, Quinn WG. 2001. Flies, genes, and learning. Annu Rev Neurosci 24: $1283-1309$

Waddell S, Armstrong JD, Kitamoto T, Kaiser K, Quinn WG. 2000. The amnesiac gene product is expressed in two neurons in the Drosophila brain that are critical for memory. Cell 103: 805-813.

Wang CY, Chang K, Petralia RS, Wang YX, Seabold GK, Wenthold RJ. 2006. A novel family of adhesion-like molecules that interacts with the NMDA receptor. J Neurosci 26: 2174-2183.

Woo J, Kwon SK, Kim E. 2009. The NGL family of leucine-rich repeat-containing synaptic adhesion molecules. Mol Cell Neurosci 42: $1-10$.

Worby CA, Simonson-Leff N, Clemens JC, Kruger RP, Muda M, Dixon JE. 2001. The sorting nexin, DSH3PX1, connects the axonal guidance receptor, Dscam, to the actin cytoskeleton. J Biol Chem 276: $41782-41789$.

Yin JC, Wallach JS, Del Vecchio M, Wilder EL, Zhou H, Quinn WG, Tully T. 1994. Induction of a dominant negative CREB transgene specifically blocks long-term memory in Drosophila. Cell 79: 49-58.

Yoshihara M, Adolfsen B, Galle KT, Littleton JT. 2005. Retrograde signaling by Syt 4 induces presynaptic release and synapse-specific growth. Science 310: 858-863.

Zhong Y, Wu CF. 1991. Altered synaptic plasticity in Drosophila memory mutants with a defective cyclic AMP cascade. Science 251: 198-201.

Zhong Y, Budnik V, Wu CF. 1992. Synaptic plasticity in Drosophila memory and hyperexcitable mutants: Role of cAMP cascade. J Neurosci 12: 644-651.

Received September 24, 2010; accepted in revised form February 10, 2011. 


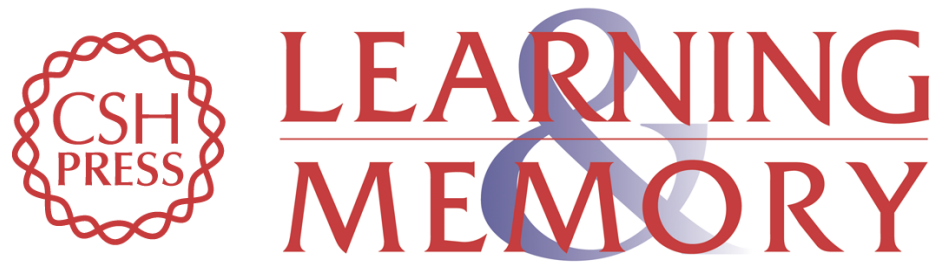

\section{Altered gene regulation and synaptic morphology in Drosophila learning and memory mutants}

Zhuo Guan, Lauren K. Buhl, William G. Quinn, et al.

Learn. Mem. 2011, 18:

Access the most recent version at doi:10.1101/lm.2027111 Supplemental
Material http://learnmem.cshlp.org/content/suppl/2011/03/21/18.4.191.DC1

References This article cites 92 articles, 35 of which can be accessed free at: http://learnmem.cshlp.org/content/18/4/191.full.html\#ref-list-1

License

Email Alerting Receive free email alerts when new articles cite this article - sign up in the box at the Service top right corner of the article or click here. 